\title{
HIGH-RESOLUTION MID-INFRARED SPECTROSCOPY OF NGC 7538 IRS 1: PROBING CHEMISTRY IN A MASSIVE YOUNG STELLAR OBJECT
}

\author{
Claudia Knez ${ }^{1,2,5}$, John H. Lacy ${ }^{1,5}$, Neal J. Evans, II ${ }^{1,5}$, Ewine F. van DishoecK ${ }^{3}$, And MatThew J. RichteR ${ }^{4,5}$ \\ ${ }^{1}$ Department of Astronomy, University of Texas at Austin, Austin, TX 78712, USA \\ ${ }^{2}$ Department of Astronomy, University of Maryland, College Park, MD 20742, USA; claudia@ astro.umd.edu \\ ${ }^{3}$ Leiden Observatory, P.O. Box 9513, 2300 RA Leiden, Netherlands \\ ${ }^{4}$ Department of Physics, University of California, Davis, One Shields Ave., Davis, CA 95616, USA \\ Received 2007 September 10; accepted 2009 January 22; published 2009 April 15
}

\begin{abstract}
We present high-resolution $(R=75,000-100,000)$ mid-infrared spectra of the high-mass embedded young star IRS 1 in the NGC 7538 star-forming region. Absorption lines from many rotational states of $\mathrm{C}_{2} \mathrm{H}_{2},{ }^{13} \mathrm{C}^{12} \mathrm{CH}_{2}$, $\mathrm{CH}_{3}, \mathrm{CH}_{4}, \mathrm{NH}_{3}, \mathrm{HCN}, \mathrm{HNCO}$, and $\mathrm{CS}$ are seen. The gas temperature, column density, covering factor, line width, and Doppler shift for each molecule are derived. All molecules were fit with two velocity components between -54 and $-63 \mathrm{~km} \mathrm{~s}^{-1}$. We find high column densities $\left(\sim 10^{16} \mathrm{~cm}^{-2}\right)$ for all the observed molecules compared to values previously reported and present new results for $\mathrm{CH}_{3}$ and $\mathrm{HNCO}$. Several physical and chemical models are considered. The favored model involves a nearly edge-on disk around a massive star. Radiation from dust in the inner disk passes through the disk atmosphere, where large molecular column densities can produce the observed absorption line spectrum.
\end{abstract}

Key words: H II regions - infrared: ISM - ISM: individual (NGC 7538 IRS 1) - ISM: molecules

Online-only material: color figures, machine-readable table

\section{INTRODUCTION}

The NGC 7538 (S158) molecular cloud, located in the Perseus arm at a distance of $2.65 \mathrm{kpc}$ (Moscadelli et al. 2009), contains many protostellar objects, making it a good candidate to study different stages of star formation. Furthermore, there seems to be a progression in evolution from northwest (the visible $\mathrm{H}$ II region, NGC 7538, and IRS 2, an only moderately obscured compact H II region) to southeast (IRS 9, a deeply embedded core) (Elmegreen \& Lada 1977; Campbell \& Thompson 1984). The near- and mid-infrared sources IRS 1, 2, and 3 all lie near the northwest end of the star-forming region. Of these, IRS 1 is the brightest mid-infrared and radio continuum source (Martin 1973; Wynn-Williams et al. 1974; Willner 1976). Its exciting star has a luminosity of $L>8 \times 10^{4} L_{\odot}$, which corresponds to a zero-age main sequence (ZAMS) spectral type earlier than O7.5, and an ionizing photon flux of $\phi>10^{48} \mathrm{~s}^{-1}$ (Werner et al. 1979; Lugo et al. 2004).

IRS 1 is embedded in a dense molecular cloud. Millimeter continuum emission is seen from the surrounding dust (Scoville et al. 1986). Pratap et al. (1989) mapped the $\mathrm{HCO}^{+}$and $\mathrm{HCN}$ emission from within $\sim 1^{\prime}$ of IRS 1 with $3^{\prime \prime}$ resolution. They found evidence for a shell-like structure on scales of 10-20" around IRS 1. Wilson et al. (1983) observed $\mathrm{NH}_{3}$ absorption toward IRS 1, and Henkel et al. (1984) mapped the absorption with the VLA. The absorption traces warm gas with a high column density of $\mathrm{NH}_{3}$ and is centered near $-60 \mathrm{~km} \mathrm{~s}^{-1}$. The emission, on the other hand, is centered near $-56.5 \mathrm{~km} \mathrm{~s}^{-1}$. The systemic velocity for this source is $\sim 57 \mathrm{~km} \mathrm{~s}^{-1}$ (van der Tak et al. 2000). More recently, Zheng et al. (2001) mapped $\mathrm{NH}_{3}$ emission from a more extended region in NGC 7538. This emission probably probes the outer envelope as indicated by the colder temperature and the smaller column density. Using

\footnotetext{
5 Visiting Astronomer at the Infrared Telescope Facility, which is operated by the University of Hawaii under contract with the National Aeronautics and Space Administration.
}

both single-dish and interferometer observations of various molecular tracers, van der Tak et al. (2000) studied the density and temperature structure of both the cold outer envelope and the warm inner material (240-72,000 AU). The inner warm region is characterized by temperatures of a few hundred Kelvin.

Outflows are often present toward protostars, and NGC 7538 IRS 1 is no exception. Campbell (1984) observed IRS 1 with the VLA at 5 and $15 \mathrm{GHz}$. She found a pair of very compact lobes of continuum radiation, separated in declination by $0 . " 2$, with emission extending out to $\pm 2^{\prime \prime}$. Her preferred model involves a bipolar ionized outflow from a late $\mathrm{O}$ star, collimated by a core of dense gas extending from $<65$ to $>25,000 \mathrm{AU}$. Further evidence of outflows was found when Gaume et al. (1995) observed IRS 1 with the VLA in the H66 $\alpha$ line and the $22 \mathrm{GHz}$ continuum. Their continuum image is similar to that of Campbell, but shows additional structure. They found broad recombination line emission in the two lobes, with a minimum line-to-continuum ratio, which they attribute to high electron density, between the lobes. They propose a model involving a high velocity stellar wind interacting with photoevaporating knots of neutral gas.

More recently, Lugo et al. (2004) have modeled the radio continuum observations of IRS 1 as due to a wind produced by photoevaporation of a circumstellar disk with a radius of $500 \mathrm{AU}$ exposed to UV radiation from the central $\mathrm{O}$ star. Maser line emission is seen from $\mathrm{H}_{2} \mathrm{O}$ (Kameya et al. 1990), OH (Dickel et al. 1982), $\mathrm{H}_{2} \mathrm{CO}$ (Hoffman et al. 2003), $\mathrm{CH}_{3} \mathrm{OH}$ (Menten et al. 1986), and $\mathrm{NH}_{3}$ (Madden et al. 1986). Minier et al. (1998) used VLBI observations of the methanol masers to infer the presence of a nearly edge-on rotating disk traced by one of the maser clusters toward IRS 1 . The central velocity of this cluster, which is the brightest cluster near IRS 1 in both $\mathrm{CH}_{3} \mathrm{OH}$ and $\mathrm{NH}_{3}$ masers, is $-56.2 \mathrm{~km} \mathrm{~s}^{-1}$. A second cluster of masers, 0 "' 25 to the south, is at $-61.0 \mathrm{~km} \mathrm{~s}^{-1}$. The disk masers lie along a line centered near the gap between the lobes of the free-free continuum radiation, although they are aligned along a position 
angle $\sim 30^{\circ}$ from the symmetry plane. Pestalozzi et al. (2004) made a detailed model of maser emission from a disk around IRS 1, supporting the suggestion of Minier et al. They concluded that the maser emission comes from disk radii of $\sim 290-750 \mathrm{AU}$. However, more recently, De Buizer \& Minier (2005) show that the $\mathrm{CH}_{3} \mathrm{OH}$ masers may trace knots in the outflow instead. Also, De Buizer \& Minier identify a disk-like structure perpendicular to the outflow direction in mid-infrared continuum. The extent of the infrared emission is $\sim 450$ AU.

Infrared absorption by dust grains and their icy mantles has also been observed toward IRS 1. Willner (1976) and Willner et al. (1982) observed $9.7 \mu \mathrm{m}$ silicate absorption and absorption by various ices, including $\mathrm{H}_{2} \mathrm{O}$. The ice bands toward IRS 1 are relatively weak, especially in comparison with NGC 7538 IRS 9, which must lie in a colder region of the NGC 7538 cloud. Gibb et al. (2004) observed the complete 2.5-20 $\mu \mathrm{m}$ region with the Short-Wavelength Spectrometer (SWS) on the Infrared Space Observatory (ISO). $\mathrm{H}_{2} \mathrm{O}, \mathrm{CO}$, and $\mathrm{CO}_{2}$ ices are clearly present, as well as other less securely identified features. Toward NGC 7538 IRS 9, $\sim 1^{\prime}$ southeast of IRS 1, a much richer ice spectrum is seen, with $\mathrm{CH}_{3} \mathrm{OH}, \mathrm{XCN}$ (probably $\mathrm{OCN}^{-}$), and $\mathrm{CH}_{4}$ also confidently identified, and $\mathrm{OCS}, \mathrm{H}_{2} \mathrm{CO}, \mathrm{HCOOH}$, and $\mathrm{NH}_{3}$ likely present.

Gas-phase absorption in the infrared has been observed toward IRS 1 by several groups. Mitchell et al. (1990) observed absorption by ${ }^{12} \mathrm{CO}$ and ${ }^{13} \mathrm{CO}$ in their $5 \mu \mathrm{m} v=1-0$ bands. They did not spectrally resolve the lines, and they used a curve of growth analysis assuming pure absorption by Gaussian lines with FWHM of $8 \mathrm{~km} \mathrm{~s}^{-1}$ to derive the gas temperature and column density from the less-saturated ${ }^{13} \mathrm{CO}$ lines. They found two absorbing components: cold gas at $25 \mathrm{~K}$, and warm gas at $176 \mathrm{~K}$. A gas density of $n_{H}>10^{6} \mathrm{~cm}^{-3}$ was required to maintain the population of the high- $J$ levels in the warm gas. Using the ISO-SWS, Lahuis \& van Dishoeck (2000) detected warmer gas than the CO observed by Mitchell et al. They observed the $\nu_{5}$ band of $\mathrm{C}_{2} \mathrm{H}_{2}$ at $13.7 \mu \mathrm{m}$ and the $\nu_{2}$ band of $\mathrm{HCN}$ at $14.0 \mu \mathrm{m}$. With the SWS resolution of $\sim 1800$, they were not able to resolve individual line shapes, and were most sensitive to the blended lines of the Q branches, but they were able to derive temperatures and column densities from the shapes of the $\mathrm{Q}$ branches and the depths of the $v=1-0$ fundamental and $v=2-1$ hot band $\mathrm{Q}$ branches. They derived $T=800 \mathrm{~K}$ and $N=0.8 \times 10^{16} \mathrm{~cm}^{-2}$ for $\mathrm{C}_{2} \mathrm{H}_{2}$, and $T=600 \mathrm{~K}$ and $N=1.0 \times 10^{16} \mathrm{~cm}^{-2}$ for $\mathrm{HCN}$. Boonman et al. (2003) derived $T=500 \mathrm{~K}$ for $\mathrm{C}_{2} \mathrm{H}_{2}$ from an updated reduction of the spectra. Boonman et al. (2003) and Boonman \& van Dishoeck (2003) studied gas absorption from $\mathrm{CO}_{2}$ and $\mathrm{H}_{2} \mathrm{O}$ using $I S O$ data. They found enhanced abundances toward the inner warm material compared to the cold envelope indicating that grain mantles are sublimating and enriching the gas-phase chemistry close to the protostar. Gas-phase $\mathrm{CH}_{4}$ was also detected toward IRS 9 (Lacy et al. 1991; Boogert et al. 2004) but not IRS 1.

The wealth of observational data available suggest the following physical scenario for NGC 7538 IRS 1 . The massive young star is surrounded by a massive cold envelope on scales 72,000 AU (van der Tak et al. 2000). At scales below $1000 \mathrm{AU}$, the temperature increases to $\geqslant 100 \mathrm{~K}$. Inside this radius, there is evidence for a disk (De Buizer \& Minier 2005) and small scale knotty outflows (Gaume et al. 1995), both of which can be affected by the UV and X-ray radiation from the protostar (e.g., Stäuber et al. 2004, 2005).

In this paper, we present high-resolution mid-infrared spectroscopy of NGC 7538 IRS 1 showing a rich absorption spectrum containing lines from seven molecules: $\mathrm{C}_{2} \mathrm{H}_{2}, \mathrm{HCN}, \mathrm{CH}_{4}, \mathrm{CH}_{3}$, $\mathrm{NH}_{3}, \mathrm{HNCO}$, and CS. Mid-infrared absorption is much more sensitive to the inner warm gas than radio observations. Previous infrared studies suffered from limited spectral resolution. Section 2 describes the observations. We then present the model used to derive the column densities and temperatures for the various species in Section 3 and describe the results for each of the molecules in Section 4. Subsequently, in Section 5, we discuss the possible scenario in which we are probing chemistry in a circumstellar disk. In Section 6, we provide some concluding remarks.

\section{OBSERVATIONS}

\subsection{Observations and Data Reduction}

The observations were made with TEXES, the Texas Echelon Cross Echelle Spectrograph (Lacy et al. 2002), on the NASA Infrared Telescope Facility (IRTF) in 2001 June and November, 2002 September and December, and 2005 December. TEXES is a high-resolution cross-dispersed spectrograph operating at mid-infrared wavelengths between 5 and $25 \mu \mathrm{m}$. It achieves a spectral resolution of $R=75,000-100,000(\Delta v=3-4 \mathrm{~km}$ $\mathrm{s}^{-1}$ ) shortward of $14 \mu \mathrm{m}$ with a slit width of $\sim 1$.'5. (Of the observations presented here, only the spectra near $11 \mu \mathrm{m}$, which used a $1^{\prime \prime}$ slit, achieved the higher resolution.) The spatial resolution along the North-South oriented slit is $\sim 1^{\prime \prime}$, slightly larger than the diffraction limit of the IRTF. The spectral and spatial sampling by the $256 \times 256$ pixel $^{2} \mathrm{Si}$ :As detector array are $1.0 \mathrm{~km} \mathrm{~s}^{-1}$ and $0 . \prime 35$, respectively. At each spectral setting, 5-10 orders of the high-resolution echelon grating are recorded, giving a spectral coverage of $\Delta \lambda_{\text {tot }} / \lambda \sim 0.5 \%$. At wavelengths shortward of $11 \mu \mathrm{m}$ full order widths are observed, giving continuous spectral coverage, but longward of $11 \mu \mathrm{m}$ the order width exceeds the array width, leaving gaps in the observed spectra. The location of the gaps can be shifted in order to optimize line coverage. However, since the gaps are small and, at $13 \mu \mathrm{m}$, there are many telluric lines, it would be impractical to repeat observations with small shifts in order to get complete spectral coverage for each setting.

Much of the data were taken in high water vapor conditions, but the observing conditions did not seriously affect the spectra except for increased noise, especially on atmospheric lines. Observations of NGC 7538 IRS 1 were interspersed with observations of a bright asteroid (usually Ceres (greater than $500 \mathrm{Jy})$, but also Hygeia $(\sim 150 \mathrm{Jy}))$, which served as a comparison source for removal of telluric absorption features. The telescope was nodded by $3-5^{\prime \prime}$, moving the source along the spectrograph entrance slit to allow subtraction of background emission. At the beginning of each set of nodded observations an ambient temperature blackbody was observed for flat-fielding. The first nods were used to peak up on the source by maximizing the throughput of the slit. The nod pairs taken during peak up were not included in the final sum.

Data reduction followed the standard TEXES procedure (Lacy et al. 2002) of first subtracting the two nod position spectra, flat-fielding with the blackbody-sky difference echellogram (Lacy et al. 1989), and interpolating over spikes and bad pixels. Optimally weighted point-source spectra were then extracted from the echellograms. The spectra were linearized in $\ln (v)$ based on the known optical distortions, and absolute wavenumber calibration was obtained from telluric emission features and corrected for the Earth's motion relative to LSR. 

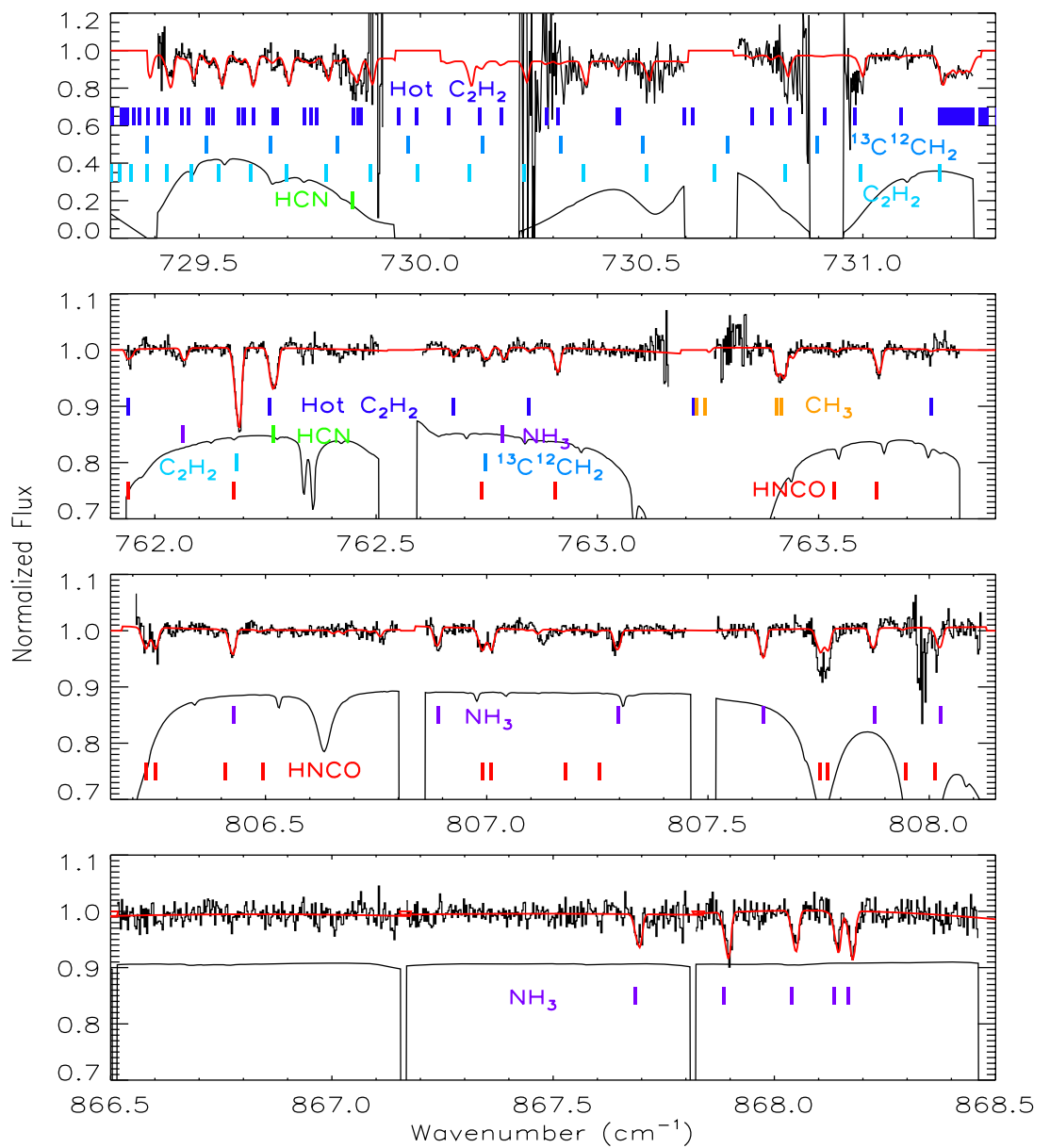

Figure 1. Selected orders (thick black) between 729 and $870 \mathrm{~cm}^{-1}$ are shown with the best fit (red). Positions of lines considered in the fit are given: $\mathrm{C}_{2} \mathrm{H}_{2}$ (light blue), ${ }^{13} \mathrm{C}^{12} \mathrm{CH}_{2}$ (blue), hot bands of $\mathrm{C}_{2} \mathrm{H}_{2}$ (dark blue), $\mathrm{HCN}$ (green), $\mathrm{CH}_{3}$ (orange), $\mathrm{HNCO}$ (red), and $\mathrm{NH}_{3}$ (purple). The thin black line indicates the atmospheric transmission. The top panel has a larger scale for the normalized flux ( $y$-axis) to show the lower atmospheric transmission. Note that there are gaps between orders.

The resulting wavenumber scale is correct to within $1 \mathrm{~km} \mathrm{~s}^{-1}$. The same procedure was used for the asteroid spectra, and then the IRS 1 spectra were divided by the asteroid spectra with correction for differences in airmass. With this procedure, telluric absorption lines as deep as $90 \%$ can be removed, although of course the noise increases on lines. However, broad response variations often remained, which were removed by fitting the continuum in each echelon order to a quartic polynomial and dividing. This procedure also sets the continuum in each order to one.

\subsection{Line Identification and New Infrared Detections}

Ten spectral settings were observed in the $728-820 \mathrm{~cm}^{-1}$ $(13.7-12.2 \mu \mathrm{m})$ region, two in the $860-930 \mathrm{~cm}^{-1}(11.5-10.7$ $\mu \mathrm{m}$ ) region (see Figure 1), and three in the $1240-1312 \mathrm{~cm}^{-1}$ $(8.0-7.6 \mu \mathrm{m})$ region (see Figure 2). A total of 110 echelon orders, or $\sim 70 \mathrm{~cm}^{-1}$, were observed. The original observations were meant to study the line profiles of $\mathrm{C}_{2} \mathrm{H}_{2}$ and $\mathrm{HCN}$, which had previously been detected. In addition to the prominent fundamental band lines of $\mathrm{C}_{2} \mathrm{H}_{2}$ and $\mathrm{HCN}$, many $\mathrm{C}_{2} \mathrm{H}_{2}$ hot band lines were detected in our spectra including lines from the $v_{4}+v_{5}-v_{4}$ and the $2 v_{5}-v_{5}$ bands. After the hot bands were identified, unidentified lines still remained, some of which were double dipped. The double lines turned out to be $\mathrm{CH}_{3}$ lines which are split due to spin-rotation interactions (see the Appendix). This is the first detection of $\mathrm{CH}_{3}$ toward dense gas and the first ground-based detection of $\mathrm{CH}_{3}$. Previously, $\mathrm{CH}_{3}$ had only been observed with ISO toward the Galactic center (Feuchtgruber et al. 2000). In efforts to detect ethane, $\mathrm{C}_{2} \mathrm{H}_{6}$, at $810-820 \mathrm{~cm}^{-1}$, many lines were detected, though none corresponded to $\mathrm{C}_{2} \mathrm{H}_{6}$. Some were due to $\mathrm{NH}_{3}$, but many lines remained unidentified (see Figures 3 and 4). Based on their separation we were able to identify the lines as due to $\mathrm{HNCO}$. Interstellar $\mathrm{HNCO}$ is a well-known hot core molecule at radio wavelengths (Zinchenko et al. 2000), but it had never been seen at infrared wavelengths. In total, five molecules were observed in the $728-820 \mathrm{~cm}^{-1}$ region: $\mathrm{C}_{2} \mathrm{H}_{2}$ (including ${ }^{13} \mathrm{C}^{12} \mathrm{CH}_{2}$ ), $\mathrm{HCN}, \mathrm{HNCO}, \mathrm{CH}_{3}$, and $\mathrm{NH}_{3}$. At higher frequencies, $\mathrm{CH}_{4}, \mathrm{C}_{2} \mathrm{H}_{2}$, and $\mathrm{CS}$ were observed in the $1240-1320 \mathrm{~cm}^{-1}$ region. $\mathrm{CH}_{4}$ observations from the ground are possible for this source because of the large Doppler shift with respect to the telluric lines.

Table 1 gives a summary of the detected lines. At least five lines were observed for each molecule, with some molecules like $\mathrm{C}_{2} \mathrm{H}_{2}$ (including isotopic lines and hot bands), and $\mathrm{NH}_{3}$ having more than 40 lines detected. However, the number of HNCO lines dominates over lines from the other molecules, with over 100 lines detected. Table 2 (full version is available online only) lists all the individual lines detected in our spectra. A selection of spectra including all observed molecules are shown in Figures 1 and 2, along with fits described in Section 3. For most molecules the line strengths were taken from the GEISA03 database. ${ }^{6}$ The

\footnotetext{
6 http://ara.lmd.polytechnique.fr
} 

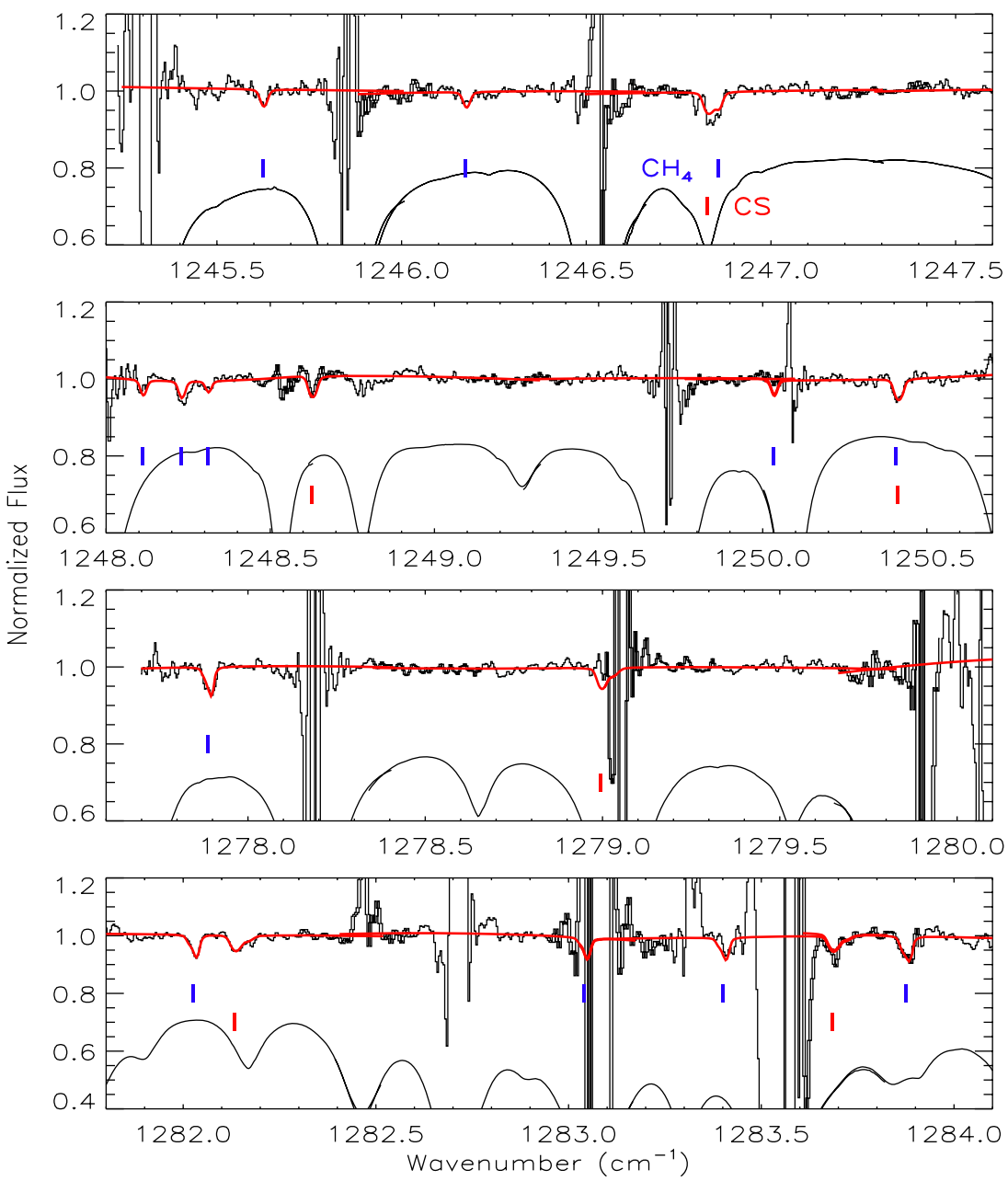

Figure 2. Selected orders (thick black) between 1245 and $1285 \mathrm{~cm}^{-1}$ are shown with the best fit (red). Position of lines considered in the fit are given: $\mathrm{CH}_{4}$ (blue) and CS (red). The thin black line indicates the atmospheric transmission. In this region there are no gaps between orders. Instead the orders overlap where the black line is darker.

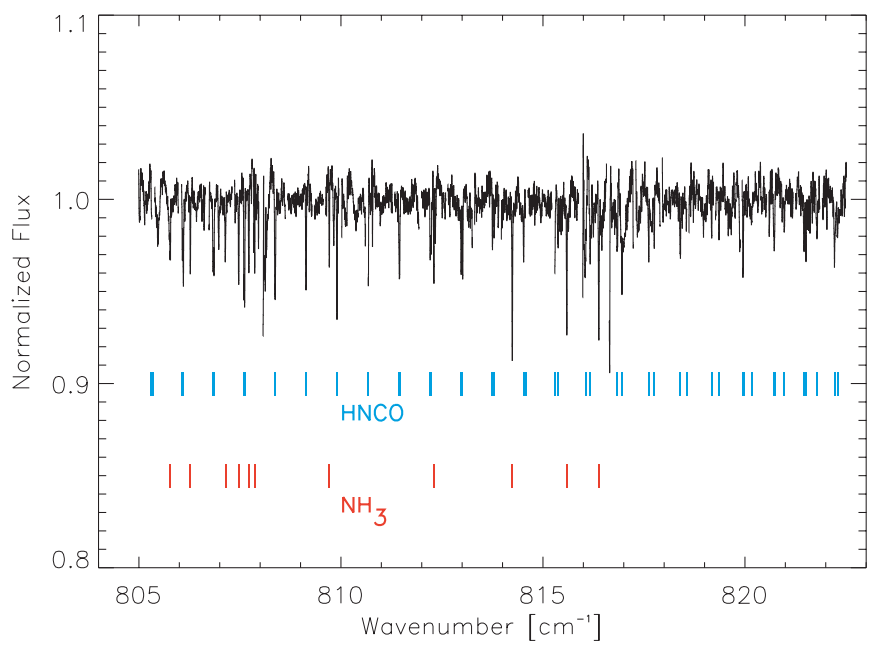

Figure 3. This figure shows a portion of spectrum containing some $\mathrm{NH}_{3}$ lines as well as a wealth of HNCO lines. These spectral settings led to the identification of HNCO. Figure 4 shows a small portion of this spectrum.

$\mathrm{CH}_{3}$ band strength was obtained from Wormhoudt \& McCurdy (1989) and the HNCO oscillator strength was obtained from Lowenthal et al. (2002). The CS oscillator strength was taken from Botschwina \& Sebald (1985).
Table 1

Summary of Observed Lines

\begin{tabular}{lcc}
\hline \hline Molecule & $\begin{array}{c}\text { Total Number of } \\
\text { Detected Lines }\end{array}$ & Band \\
\hline $\mathrm{C}_{2} \mathrm{H}_{2}$ & 25 & $v_{5}$ \\
$\mathrm{C}_{2} \mathrm{H}_{2}{ }^{\mathrm{a}}$ & 2 & $v_{4}+v_{5}$ \\
$\mathrm{C}_{2} \mathrm{H}_{2}{ }^{\mathrm{b}}$ & 20 & $v_{4}+v_{5}-v_{4}$ \\
${ }^{13} \mathrm{C}^{12} \mathrm{CH}_{2}{ }^{\mathrm{c}}$ & 7 & $v_{5}$ \\
$\mathrm{HCN}^{\mathrm{H}}$ & 10 & $v_{2}$ \\
$\mathrm{NH}_{3}$ & 64 & $v_{2}$ \\
$\mathrm{HNCO}$ & 125 & $v_{4}$ \\
$\mathrm{CH}_{3}$ & 11 & $v_{2}$ \\
$\mathrm{CH}_{4}$ & 12 & $v_{2} / v_{4}$ dyad \\
$\mathrm{CS}$ & 6 & $v_{2}$ \\
\hline
\end{tabular}

Notes.

a These lines were observed at $8 \mu \mathrm{m}$.

${ }^{\mathrm{b}}$ Lines in Q-branch at $731 \mathrm{~cm}^{-1}$. See the top panel of Figure 1.

${ }^{\mathrm{c}}$ Only unblended lines are reported here.

\section{SPECTRUM FITTING}

To derive physical parameters of the absorbing gas, model spectra were fitted to the observations. For this purpose, the data were divided into two groups. All data at wavenumbers between 700 and $1000 \mathrm{~cm}^{-1}(10.7-13.7 \mu \mathrm{m})$ were fitted simultaneously, 


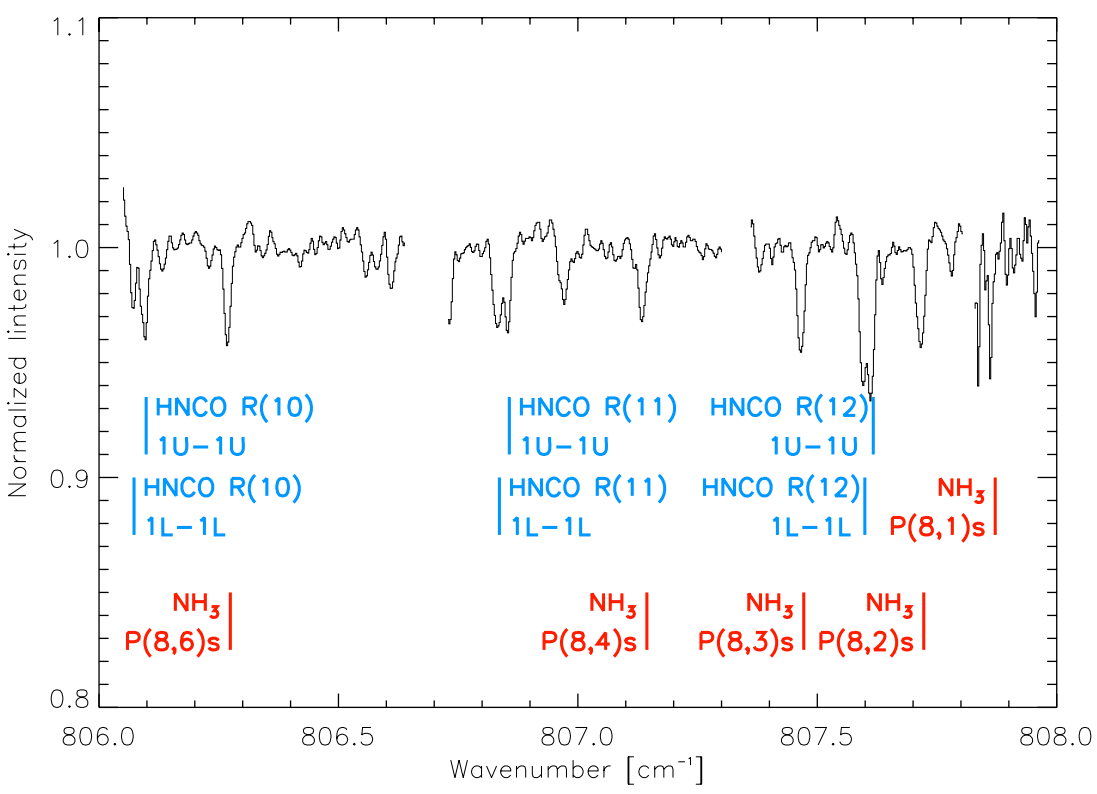

Figure 4. Profiles of several $\mathrm{HNCO}$ and $\mathrm{NH}_{3}$ lines in the spectral region shown in Figure 3. The two HNCO subbands shown are R-branch transitions where the $K=1$ state is split into upper $(\mathrm{U})$ and lower $(\mathrm{L})$ levels. Because of the close separation the subbands, the lines can overlap for a range of J. See the Appendix for more details on transition rules for HNCO.

(A color version of this figure is available in the online journal.)

Table 2

Observed Lines

\begin{tabular}{lcccc}
\hline \hline Molecule & Line & $\begin{array}{c}\text { Wavenumber } \\
\left(\mathrm{cm}^{-1}\right)\end{array}$ & $\begin{array}{c}E_{J} \\
(\mathrm{~K})\end{array}$ & Band \\
\hline $\mathrm{C}_{2} \mathrm{H}_{2}$ & $\mathrm{Q}(5)$ & 729.289 & 35.30 & $\nu_{5}$ \\
$\mathrm{C}_{2} \mathrm{H}_{2}$ & $\mathrm{Q}(6)$ & 729.343 & 49.42 & $\nu_{5}$ \\
$\mathrm{C}_{2} \mathrm{H}_{2}$ & $\mathrm{Q}(7)$ & 729.406 & 65.89 & $\nu_{5}$ \\
$\mathrm{C}_{2} \mathrm{H}_{2}$ & $\mathrm{Q}(8)$ & 729.477 & 84.71 & $\nu_{5}$ \\
$\mathrm{C}_{2} \mathrm{H}_{2}$ & $\mathrm{Q}(9)$ & 729.558 & 105.89 & $\nu_{5}$ \\
$\mathrm{C}_{2} \mathrm{H}_{2}$ & $\mathrm{Q}(10)$ & 729.648 & 129.41 & $\nu_{5}$ \\
$\mathrm{C}_{2} \mathrm{H}_{2}$ & $\mathrm{Q}(11)$ & 729.747 & 155.29 & $\nu_{5}$ \\
$\mathrm{C}_{2} \mathrm{H}_{2}$ & $\mathrm{Q}(14)$ & 730.096 & 247.02 & $\nu_{5}$ \\
$\mathrm{C}_{2} \mathrm{H}_{2}$ & $\mathrm{Q}(15)$ & 730.230 & 282.30 & $\nu_{5}$ \\
$\mathrm{C}_{2} \mathrm{H}_{2}$ & $\mathrm{Q}(16)$ & 730.373 & 319.93 & $\nu_{5}$ \\
$\mathrm{C}_{2} \mathrm{H}_{2}$ & $\mathrm{Q}(18)$ & 730.686 & 402.22 & $\nu_{5}$ \\
$\mathrm{C}_{2} \mathrm{H}_{2}$ & $\mathrm{Q}(19)$ & 730.855 & 446.89 & $\nu_{5}$ \\
$\mathrm{C}_{2} \mathrm{H}_{2}$ & $\mathrm{Q}(20)$ & 731.034 & 493.90 & $\nu_{5}$ \\
$\mathrm{C}_{2} \mathrm{H}_{2}$ & $\mathrm{Q}(26)$ & 732.287 & 825.20 & $\nu_{5}$ \\
$\mathrm{C}_{2} \mathrm{H}_{2}$ & $\mathrm{Q}(27)$ & 732.526 & 888.62 & $\nu_{5}$ \\
\hline
\end{tabular}

Notes. Only lines that were individually detected at the $3 \sigma$ level are included in this list.

(This table is available in its entirety in a machine-readable form in the online journal. A portion is shown here for guidance regarding its form and content.)

and data at $1240-1280 \mathrm{~cm}^{-1}$ (near $8 \mu \mathrm{m}$ ) were fitted separately. The Marquardt fitting procedure (Bevington \& Robinson 2003) was used, which minimizes $\chi^{2}$, the summed squared deviation normalized by the squared noise, between the data and the model.

Fitting was first attempted with a single component for each molecule, but the residuals suggested that a better fit could be obtained with multiple velocity components. The model that we used assumed that each molecule is found in one or two absorbing components. Each component is described by its Doppler shift, $V_{\mathrm{LSR}}$, its Gaussian line width (1/e half width $\left.=\left(2 \mathrm{kT} \mathrm{m}^{-1}\right)^{1 / 2}\right), b$, the molecule's column density, $N$, the rotational temperature of the gas, $T$, and the covering factor, $C$ (the fraction of the background continuum source covered

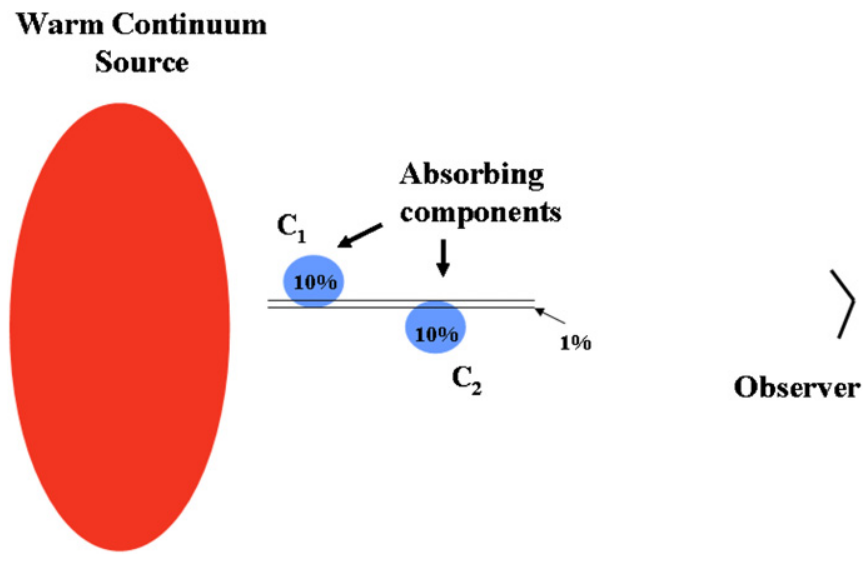

Figure 5. Two absorbing components between the continuum source and the observer. Each component covers $10 \%$ of the continuum source. The overlap of the two absorbing regions is given by the product of their covering factor. In this case, only $1 \%$ of the continuum source is covered by both components.

(A color version of this figure is available in the online journal.)

by the component). The fitted column density is the average over the partially covered source, so the column density in the covered portion would be $N / C$. A covering factor of, e.g., $10 \%$ means that lines saturate at $90 \%$ of the continuum flux and the column density in the absorbing component is ten times the average over the continuum source. The effect modeled in this way could also result from veiling (continuum emission from foreground or surrounding material) or from re-emission in the lines by the absorbing molecules. The components were assumed to overlap by the products of their covering factors (see Figure 5). The following equation was used to calculate the observed transmission:

$$
I_{\mathrm{obs}}=I_{0}\left(1-C_{1}\left(1-e^{-\tau_{1}(v)}\right)\right)\left(1-C_{2}\left(1-e^{-\tau_{2}(\nu)}\right)\right),
$$

where $C_{1}$ and $C_{2}$ are the covering factors for the two components. This results in a greater absorption in saturated lines than would result if the optical depths were added first and then the 
Table 3

Results from the Model Fit to the Spectra

\begin{tabular}{|c|c|c|c|c|c|c|c|c|c|c|}
\hline \multirow[t]{2}{*}{ Molecule } & \multicolumn{5}{|c|}{ Component 1} & \multicolumn{5}{|c|}{ Component 2} \\
\hline & $\begin{array}{c}V_{\mathrm{LSR}} \\
\left(\mathrm{km} \mathrm{s}^{-1}\right)\end{array}$ & $\begin{array}{c}b \\
\left(\mathrm{~km} \mathrm{~s}^{-1}\right) \\
\end{array}$ & $\begin{array}{l}T_{\mathrm{ex}} \\
(\mathrm{K})\end{array}$ & $\begin{array}{c}N(X) \\
\left(10^{16} \mathrm{~cm}^{-2}\right) \\
\end{array}$ & $\begin{array}{c}\mathrm{C} \\
(\times 100)\end{array}$ & $\begin{array}{c}V_{\mathrm{LSR}} \\
\left(\mathrm{km} \mathrm{s}^{-1}\right)\end{array}$ & $\begin{array}{c}b \\
\left(\mathrm{~km} \mathrm{~s}^{-1}\right) \\
\end{array}$ & $\begin{array}{l}T_{\mathrm{ex}} \\
(\mathrm{K})\end{array}$ & $\begin{array}{c}N(X) \\
\left(10^{16} \mathrm{~cm}^{-2}\right) \\
\end{array}$ & $\begin{array}{c}\mathrm{C} \\
(\times 100)\end{array}$ \\
\hline$\overline{\mathrm{C}_{2} \mathrm{H}_{2}}$ & $-55.7(0.3)$ & $0.6(0.1)$ & $225(20)$ & $3.0(0.6)$ & $5.9(0.6)$ & $-59.4(0.3)$ & $0.6(0.1)$ & $191(10)$ & $2.8(0.2)$ & $23.7(1.0)$ \\
\hline $\mathrm{HNCO}$ & $-57.2(0.3)$ & $1.8(0.2)$ & $319(27)$ & $0.4(0.3)$ & $3.8(0.3)$ & $-60.2(0.2)$ & $0.7(0.4)$ & $171(79)$ & $0.1(0.3)$ & $97.0(11)$ \\
\hline $\mathrm{CH}_{3}$ & $-54.2(0.4)$ & $1.8(0.6)$ & $258(17)$ & $1.7(0.2)$ & $97.0(15)$ & $-62.8(0.5)$ & $1.5(0.9)$ & 927 (161) & $0.6(0.1)$ & $97.3(18)$ \\
\hline $\mathrm{NH}_{3}$ & $-57.3(0.3)$ & $0.9(1.0)$ & $278(31)$ & $5.2(1.5)$ & $8.9(1.2)$ & $-60.1(0.2)$ & $0.4(0.1)$ & $248(32)$ & $2.8(0.8)$ & $15.8(3.6)$ \\
\hline
\end{tabular}

Notes. The column densities listed are $N_{\text {tot }} \times C$.

transmission spectrum was calculated from the optical depth spectrum. This approach was chosen because it gave a better fit to the data than adding the optical depths first and assuming the same covering factor, but it assumes that different components absorb along different lines of sight to a partially covered continuum source, which may not be the case.

A frequency correction was allowed for each spectral setting observed, to correct for errors in wavelength calibration. With only one exception, the correction was less than a $1 \mathrm{~km}$ $\mathrm{s}^{-1}$ Doppler shift. In addition, a broadening of the instrumental resolution was permitted in the fitting to allow for imperfect internal instrument focusing, or possibly a "macroturbulent" broadening in the absorbing gas. The fitting program chose a resolution a factor of 1.2 greater than that derived from gas cell data. A constant frequency resolution (as opposed to a constant Doppler resolution) was used in each of the two fitted regions. This represents well the improving resolving power toward shorter wavelengths in the 10.7-13.7 $\mu \mathrm{m}$ region. In addition to these parameters, the continuum, slope, and curvature of each echelon order were varied, to allow correction of the baseline fitting done during data reduction. The continuum fitting and frequency correction required about 300 free, but rather easily determined, parameters. Fewer parameters are needed to determine the physical conditions such as temperature, column density, line width and covering factor: 70 parameters for the $10.7-13.7 \mu \mathrm{m}$ spectra and 21 for the $8 \mu \mathrm{m}$ region. For the $10-13 \mu \mathrm{m}$ region, we have over 3000 points to constrain the 70 parameters of interest if we characterize the constraining points by the number of lines times the number of pixels for each line.

For $\mathrm{C}_{2} \mathrm{H}_{2}$ and $\mathrm{HNCO}$, lines of several bands were observed. For $\mathrm{C}_{2} \mathrm{H}_{2}$, in addition to lines of the $\mathrm{Q}$ and $\mathrm{R}$ branches of the $v_{5}$ fundamental, lines of several $v_{4}+v_{5}-v_{5}$ and $2 v_{5}-v_{5}$ bands (involving absorption from the excited $v_{4}$ and $v_{5}$ vibration states) were observed. The vibrational temperatures describing the populations of the $v_{4}$ and $v_{5}$ states were included as free parameters in the fit. R-branch lines of the $\nu_{5}$ band of ${ }^{13} \mathrm{C}^{12} \mathrm{CH}_{2}$ were also observed, and the ${ }^{13} \mathrm{C}^{12} \mathrm{CH}_{2} / \mathrm{C}_{2} \mathrm{H}_{2}$ abundance ratio was allowed to vary, although it was kept the same in all the $\mathrm{C}_{2} \mathrm{H}_{2}$ components. Two $\mathrm{C}_{2} \mathrm{H}_{2}$ lines of the $v_{4}+v_{5}$ band at $7.6 \mu \mathrm{m}$ were observed as well. For HNCO, lines from the $v_{4}$ band were observed. HNCO is an only slightly nonlinear molecule, and its spectrum consists of a series of subbands resembling those of a linear molecule, like $\mathrm{C}_{2} \mathrm{H}_{2}$. Its dipole moment oscillates diagonally, giving it a-type subbands, with no change in the angular momentum about its long axis and only $\mathrm{P}$ and $\mathrm{R}$ branches, and $\mathrm{b}$-type subbands, in which the angular momentum about its long axis changes, and $\mathrm{P}, \mathrm{Q}$, and $\mathrm{R}$ branches are seen. See the Appendix for further discussion of the HNCO spectrum. Since we did not have laboratory data regarding the relative strengths of the different subbands, we considered them to be caused by different species and summed the abundances to obtain the $\mathrm{HNCO}$ abundance. The $\mathrm{HCN}$ lines observed were from the $v_{2}$ bending mode. We included HCN $2 v_{2}-v_{2}$ lines in the fit. Although no hot HCN lines were obvious in the spectra, their inclusion lowered $\chi^{2}$ significantly $\left(\Delta \chi^{2}=13\right)$ and changed the best-fitting $N_{\mathrm{HCN}}$ noticeably.

Although it might not be apparent from the spectra, given the shallowness of the lines, many of the detected lines are at least moderately saturated, and some are very saturated. The lines only appear weak because of the small covering factors (or emission filling in the absorption lines) and to a lesser extent the small line widths. This conclusion is very robust; the relative depths of lines of different opacity requires substantial saturation. This is most apparent in the near equality of the depths of the ortho and para lines in the $\mathrm{C}_{2} \mathrm{H}_{2} \mathrm{Q}$ branch, near $730 \mathrm{~cm}^{-1}$ (see the top panel of Figure 1). HCN in the $-60 \mathrm{~km} \mathrm{~s}^{-1}$ component and $\mathrm{NH}_{3}$ in the $-56.5 \mathrm{~km} \mathrm{~s}^{-1}$ component are so saturated that only lower limits could be placed on their abundances until their isotopomers were included in the fit to provide upper limit constraints. By including isotopomers and intrinsically weak lines, a wide enough range of line optical depths was observed to allow meaningful constraints to be placed on the molecular abundances in spite of the saturation.

The observed lines of $\mathrm{CH}_{3}$ were in the $\mathrm{R}$ branch of the $v_{2}$ outof-plane bending mode of this planar molecule. Each of these lines is doubled by the interaction between the electron spin and the molecular rotation (see the Appendix). The $\mathrm{CH}_{3} v_{2}$ band center is at $16.5 \mu \mathrm{m}$, whereas all observed lines were shortward of $13.5 \mu \mathrm{m}$, with $J \geqslant 7$, making us rather insensitive to cold $\mathrm{CH}_{3}$. Lines of the $\mathrm{P}$ and $\mathrm{Q}$ branches of the $\mathrm{NH}_{3} v_{2}$ band were observed. There was no evidence of ${ }^{15} \mathrm{NH}_{3}$ lines, but they were included in the fit to constrain the $\mathrm{NH}_{3}$ abundance, as was done with $\mathrm{H}^{13} \mathrm{CN}$. In the $8 \mu \mathrm{m}$ region, which was fitted separately, lines of the $v_{2} / v_{4}$ dyad of $\mathrm{CH}_{4}$ were observed. Because of the strong absorption by low- $J$ lines of $\mathrm{CH}_{4}$ in the Earth's atmosphere, most of the observed lines originate at $J>4$. We were able to observe the $R(0)$ line due to the favorable Doppler shift of the source $\left(\sim-57 \mathrm{~km} \mathrm{~s}^{-1}\right)$. The $\mathrm{R}(0)$ line helps constrain the temperature derived from the high $J$ lines. We also detected and fitted absorption by CS.

The derived component parameters are given in Table 3 . Uncertainties for the parameters, given in parentheses, are three times the square roots of the diagonal elements of the error 
matrix, which are uncertainties allowing all other parameters to vary. The noise in the fitted spectra is assumed to be purely photon statistical noise. With this noise estimate, the reduced $\chi^{2} \approx 1.6$, indicating that nonstatistical noise sources contribute moderately, or perhaps that a different model for the line profiles is justified. Including nonstatistical noise, the uncertainties given might reasonably be taken to be $95 \%$ confidence intervals. The larger fractional errors are asymmetric, and would be more symmetric in the log of the parameters. For column densities, this is a result of the nonlinearity of the curve of growth for moderately saturated lines. Column densities with errors greater than their values are not consistent with zero. For unsaturated lines, the derived covering factors are very uncertain because equivalent widths depend only on the product of the covering factor and the column density. For example, the column densities of $\mathrm{HNCO}$ and $\mathrm{CH}_{3}$ have smaller fractional uncertainties than those of $\mathrm{C}_{2} \mathrm{H}_{2}$ and $\mathrm{HCN}$, whereas the covering factors have smaller uncertainties for $\mathrm{C}_{2} \mathrm{H}_{2}$ and $\mathrm{HCN}$. All of the fitted molecules are detected with very high confidence.

\section{RESULTS}

For most molecules, we found two components with Doppler shifts of $V_{\mathrm{LSR}}=-56.4 \pm 0.3$ and $-59.7 \pm 0.3 \mathrm{~km} \mathrm{~s}^{-1}$, with widths of $b=1-2 \mathrm{~km} \mathrm{~s}^{-1}$ (see Table 3). Most of the component temperatures were in the range $200-400 \mathrm{~K}$ and covering factors were typically $0.04-0.08$, with $\mathrm{C}_{2} \mathrm{H}_{2}$ requiring somewhat larger values of 0.06 and 0.18 . $\mathrm{HNCO}$ has somewhat broader component line widths but other parameters are similar. $\mathrm{CH}_{3}$ has the broadest component line widths, with a greater velocity spacing between the two components and the hottest component. Both $\mathrm{CS}$ and $\mathrm{CH}_{3}$ are fitted with much larger covering factors (consistent with 100\%) than were the other molecules. However, these covering factors are quite uncertain due to the weakness of the observed lines, and the large values may not be significant. The model spectra resulting from the best fit parameters can be seen in Figures 1 and 2.

In addition to agreeing among the different molecules (except $\mathrm{CH}_{3}$ ), the Doppler shifts agree with the two most commonly observed values for molecules seen at radio wavelengths. This gives us some confidence that the two velocity components, at -56.5 and $-60 \mathrm{~km} \mathrm{~s}^{-1}$, are real, although whether they are two separate components, or just a way of describing a nonGaussian velocity distribution is difficult to determine from the data. Generally, the temperatures of the two components for a given molecule are not very different, although a fit constraining them to be equal was significantly worse than the fit allowing all temperatures to vary. Line widths, column densities, and covering factors vary more. The fit was very poor when they were constrained to be equal for all molecules.

Note that our quoted column densities are averages over the lines of sight to the continuum emitting material, or column densities in the absorbing gas multiplied by the covering factors. There are several possible interpretations for the covering factor parameters: the continuum source could in fact be partially covered, foreground or surrounding emission could veil the spectrum, or re-emission in the lines could fill in the absorption. In all of these cases, the column densities through the absorbing material are given by $\mathrm{N} / \mathrm{C}$, rather than $\mathrm{N}$, the average column density over the line of sight in the partial covering interpretation. Consequently, if we could determine the $\mathrm{H}_{2}$ column density along the lines of sight on which we see absorption we should compare that to our N/C values.
Table 4

Abundances with Respect to $\mathrm{CO}$ and $\mathrm{H}_{2}$

\begin{tabular}{lcc}
\hline \hline Molecule & $\begin{array}{c}N(\mathrm{X}) / N(\mathrm{CO})^{\mathrm{a}} \\
\left(10^{-3}\right)\end{array}$ & $\begin{array}{c}N(\mathrm{X}) / N\left(\mathrm{H}_{2}{ }^{\mathrm{a}}\right. \\
\left(10^{-7}\right)\end{array}$ \\
\hline $\mathrm{C}_{2} \mathrm{H}_{2}$ & 5.7 & 7.7 \\
$\mathrm{HCN}$ & 6.9 & 9.2 \\
$\mathrm{HNCO}$ & 0.5 & 0.6 \\
$\mathrm{CH}_{3}$ & 2.3 & 3.1 \\
$\mathrm{NH}_{3}$ & 8.1 & 10.8 \\
$\mathrm{CH}_{4}$ & 36 & 48 \\
$\mathrm{CS}$ & 7.0 & 9.3 \\
\hline
\end{tabular}

Notes. ${ }^{a} N(\mathrm{CO})=1.0 \times 10^{19} \mathrm{~cm}^{-2}$ and $N\left(\mathrm{H}_{2}\right)=7.5 \times 10^{22} \mathrm{~cm}^{-2}$. See the text for discussion of whether these molecules are measured on the same lines of sight as the ones we observe. We use $N$ listed in Table 3.

\section{1. $\mathrm{H}_{2}$ and $\mathrm{CO}$ From Previous Measurements}

Before discussing the results of our measurements of individual molecules, we attempt to derive $\mathrm{H}_{2}$ and $\mathrm{CO}$ column densities from previous measurements. The $\mathrm{H}_{2}$ column toward NGC 7538 IRS 1 has not been directly measured, but it may be estimated from the extinction measurements of Willner (1976) with the assumption of a normal interstellar gas-to-dust ratio. This method gives $\mathrm{N}_{\mathrm{H}_{2}}=6-9 \times 10^{22} \mathrm{~cm}^{-2}$. If the continuum source is nonuniformly covered, this column density represents an average over the source. Hence, it should probably be compared to the values we derive for average column densities, $N$, of the molecules we observe. However, the silicate optical depth includes extinction from all material along the line of sight to NGC 7538, most of which is unlikely to contain our molecules. This suggests that $6-9 \times 10^{22} \mathrm{~cm}^{-2}$ is an overestimate of $\mathrm{N}_{\mathrm{H}_{2}}$ in the relevant gas. On the other hand, we suggest below that we may be observing absorption by gas in the atmosphere of a protoplanetary disk, and dust in such a region could be depleted by settling toward the disk midplane. This effect would result in an underestimate of $\mathrm{N}_{\mathrm{H}_{2}}$ from the extinction. We give the ratios of our derived column densities to an $\mathrm{H}_{2}$ column density of $7.5 \times 10^{22} \mathrm{~cm}^{-2}$ in Table 4 .

It may be preferable to compare our column densities to the column density of $\mathrm{CO}$, which has been observed in absorption toward NGC 7538 IRS 1 by Mitchell et al. (1990). They obtain a column density of $1.5 \pm 0.3 \times 10^{17} \mathrm{~cm}^{-2}$ for ${ }^{13} \mathrm{CO}$ in cold gas at $25 \mathrm{~K}$ and $1.4 \pm 0.1 \times 10^{17} \mathrm{~cm}^{-2}$ in warm gas at $176 \mathrm{~K}$. We would not be sensitive to their cold gas. Presumably their warm gas includes both of our components, although our temperatures are generally higher than theirs. If we assume ${ }^{12} \mathrm{CO} /{ }^{13} \mathrm{CO}=72$, using the ${ }^{12} \mathrm{C} /{ }^{13} \mathrm{C}$ ratio we derived from our $\mathrm{C}_{2} \mathrm{H}_{2}$ observations, we derive the abundances of our molecules relative to $\mathrm{CO}$ listed in Table 4. (Note that Mitchell et al. use ${ }^{12} \mathrm{C} /{ }^{13} \mathrm{C}=89$.) However, we should note that we have measured one spectral setting including CO lines, while searching for OCS absorption near $5 \mu \mathrm{m}$. The noise is rather high, but it is apparent that the ${ }^{13} \mathrm{CO}$ lines are not well described by moderately saturated Gaussians, as assumed by Mitchell et al. The ${ }^{13} \mathrm{CO}$ lines are deeper and broader than the lines of any of our other molecules, and have prominent blue-shifted shoulders probably tracing an outflow. We suspect that the lines are more saturated, and the $\mathrm{CO}$ column density larger than Mitchell et al. concluded. The different shapes and greater depths of the $\mathrm{CO}$ lines than our $\mathrm{C}_{2} \mathrm{H}_{2}$ lines also indicates that $\mathrm{CO}$ probes different gas. Likely, $\mathrm{C}_{2} \mathrm{H}_{2}$ and the other molecules we observe in the mid-infrared are found only in unusual regions, whereas $\mathrm{CO}$ is distributed along the entire line of sight. 


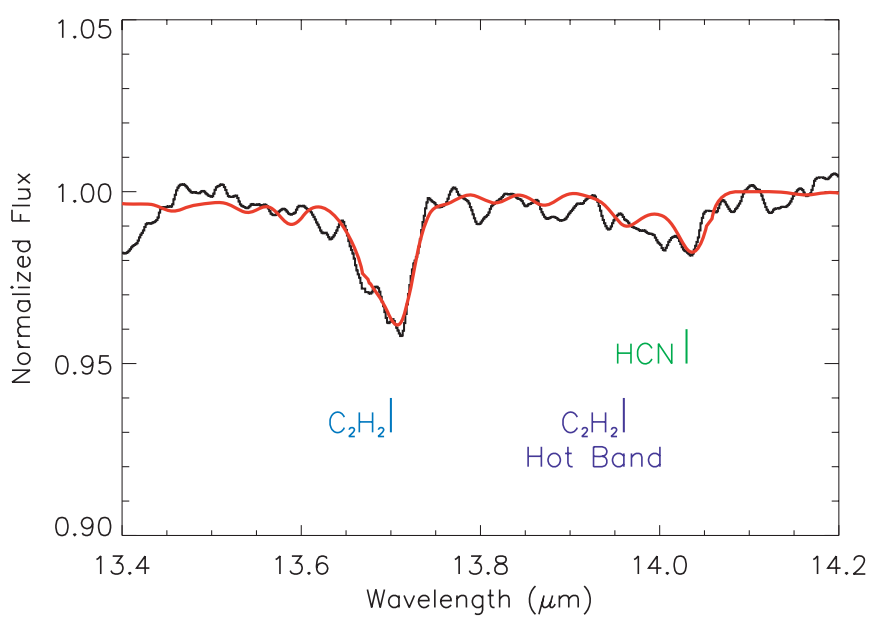

Figure 6. Spectrum of NGC 7538 IRS 1 as seen by ISO is shown in black. A fit to the $\mathrm{C}_{2} \mathrm{H}_{2}$ and $\mathrm{HCN}$ fundamental Q-branches as well as a hot band Q-branch of $\mathrm{C}_{2} \mathrm{H}_{2}$ is shown in grey. The parameters are set from the TEXES high-resolution observations. The models gives a good fit to the ISO spectrum.

(A color version of this figure is available in the online journal.)

In general, we conclude that the ratios of column densities to those of either $\mathrm{H}_{2}$ or $\mathrm{CO}$ given in Table 4 are quite uncertain because the different column density tracers may be sensitive to different components along the line of sight. The column densities of the different molecules we observe should be much more comparable.

\section{2. $\mathrm{C}_{2} \mathrm{H}_{2}$ and $\mathrm{HCN}$}

Since our initial search for molecules began with $\mathrm{C}_{2} \mathrm{H}_{2}$ and $\mathrm{HCN}$, the first models only included those two molecules. In these models, we derived similar temperatures and column densities as derived from ISO observations (Lahuis \& van Dishoeck 2000; Boonman et al. 2003). Once we include absorption from other molecules, $\mathrm{HNCO}$ in particular, the temperatures for $\mathrm{HCN}$ and especially for $\mathrm{C}_{2} \mathrm{H}_{2}$ are lowered significantly. Some HNCO lines overlap high- $J$ lines of $\mathrm{C}_{2} \mathrm{H}_{2}$, which means that more hot $\mathrm{C}_{2} \mathrm{H}_{2}$ is necessary to produce the observed absorption when $\mathrm{HNCO}$ is not included in the model. In our final model including all of the observed molecules, our derived column densities of $\mathrm{C}_{2} \mathrm{H}_{2}, N=$ $5.8 \times 10^{16} \mathrm{~cm}^{-2}$, and HCN, $N=6.9 \times 10^{16} \mathrm{~cm}^{-2}$, (see Table 3) are larger than those derived in Lahuis \& van Dishoeck, $N_{\mathrm{C}_{2} \mathrm{H}_{2}}=8 \times 10^{15} \mathrm{~cm}^{-2}$ and $N_{\mathrm{HCN}}=1.0 \times 10^{16} \mathrm{~cm}^{-2}$. Part of the difference is due to the different Doppler parameter used: $b=5 \mathrm{~km} \mathrm{~s}^{-1}$ is used in Lahuis \& van Dishoeck, whereas, we derive $b \sim 1 \mathrm{~km} \mathrm{~s}^{-1}$. We derive lower temperatures, $T_{\mathrm{C}_{2} \mathrm{H}_{2}}=190-230 \mathrm{~K}$ and $T_{\mathrm{HCN}}=250-450 \mathrm{~K}$ than derived by Lahuis \& van Dishoeck, $T_{\mathrm{C}_{2} \mathrm{H}_{2}}=800 \mathrm{~K}$ and $T_{\mathrm{HCN}}=600 \mathrm{~K}$, and Boonman et al. (2003), $T_{\mathrm{C}_{2} \mathrm{H}_{2}}=500 \mathrm{~K}$. We also find that we need a small covering factor to account for the saturated yet shallow lines. The level of saturation is difficult to determine from the ISO data. In order to verify that our fit to the TEXES data is also consistent with the $I S O$ observations, we take our model at the ISO-SWS resolution and compare to the data (Figure 6). The model matches the fundamental Q-branches for the two molecules as well as a hot band Q-branch of $\mathrm{C}_{2} \mathrm{H}_{2}$.

$\mathrm{C}_{2} \mathrm{H}_{2}$ is expected to be in rotational local thermal equilibrium (LTE) since it has no permanent dipole moment. The fact that the two excited vibrational states of $\mathrm{C}_{2} \mathrm{H}_{2}$ from which absorption was observed, $v_{4}$ and $v_{5}$, are found to be populated near LTE at the $\mathrm{C}_{2} \mathrm{H}_{2}$ rotational temperature, or even slightly higher, provides information about the physical conditions of the gas. For both bands, the hot bands indicate they are slightly out of LTE since the vibrational temperatures $\left(T_{v}\right)$ are higher than the rotational temperatures $\left(T_{r}\right)$. For the $v_{4}+v_{5}-v_{4}$ bands, the $T_{v}$ temperatures for the two components were 346 and $271 \mathrm{~K}$ while for the $2 v_{5}-v_{5}$ bands, they were 277 and $227 \mathrm{~K}$. The rotational temperatures for the $v_{5}$ fundamental are 225 and $191 \mathrm{~K}$ for the two components. In LTE, the vibrational temperatures for the hot bands would equal the rotational temperature measured by the fundamental band lines.

The $v_{4}$ level has no allowed radiative transitions to the ground vibrational level, and none of the rotational levels of the ground vibrational level are coupled radiatively, in both cases due to the symmetry of the $\mathrm{C}_{2} \mathrm{H}_{2}$ molecule. This suggests that the populations of the ground and $v_{4}$ states should be set by collisions, and so should be in LTE at the same temperature. However, the $v_{4}$ level can be radiatively pumped by absorption in the $7.6 \mu \mathrm{m} \nu_{4}+v_{5}$ band followed by emission of photons in the $v_{4}+v_{5}-v_{4}$ band. The $v_{5}$ state must be populated predominantly radiatively for any plausible density, since the critical density is $>10^{10} \mathrm{~cm}^{-3}$. From these considerations, we conclude that the brightness temperature of the radiation field seen by the observed gas must be close to the kinetic temperature of the gas. In addition, the fact that the measured rotational temperature for $\mathrm{HCN}$ is similar to that of $\mathrm{C}_{2} \mathrm{H}_{2}$ indicates that the rotational levels of $\mathrm{HCN}$ are kept populated either by collisions, which would require a gas density $>10^{7} \mathrm{~cm}^{-3}$, or by infrared pumping through the $v_{2}$ transitions. Lahuis \& van Dishoeck (2000) conclude that either excitation mode is possible. The presence of $\mathrm{C}_{2} \mathrm{H}_{2} 2 v_{5}-v_{5}$ absorption requires infrared pumping, although $v_{4}+v_{5}-v_{4}$ absorption does not.

We can attempt to estimate the extent to which emission in our lines fills in the absorption, at least for $\mathrm{C}_{2} \mathrm{H}_{2}$, by using the $2 v_{5}$ $\nu_{5}$ lines as probes of the $\nu_{5}$ population. Our spectra require a $\nu_{5}$ vibrational temperature $\sim 300 \mathrm{~K}$. We need to compare this number to the blackbody brightness temperature of the background continuum radiation. From the shape of the 8$13 \mu \mathrm{m}$ spectrum, Willner derived a dust temperature of $330 \mathrm{~K}$ assuming the emitting dust is optically thick, or $370 \mathrm{~K}$ assuming optically thin silicate emission. He preferred the latter model, which gives a brightness temperature only when combined with an assumption about the solid angle subtended by the source. In either case, our vibrational temperature is only $\sim 10 \%$ less than the background temperature, so that the source function is $\sim 30 \%$ below the background intensity, and lines would be expected to saturate at $\sim 70 \%$ of the continuum. However, the continuum spectrum from which Willner derived a dust temperature is the average over the continuum source. If the gas we observe covers only a small fraction of the source, the brightness temperature of the radiation passing through that gas could be much larger than the average.

Given the many assumptions and uncertainties in this calculation, it is difficult to make a strong statement. The level at which our $\mathrm{C}_{2} \mathrm{H}_{2}$ lines saturate, $\sim 85 \%$ of the continuum, which we model with a small covering factor, could instead be due partly or entirely to emission by the absorbing gas. It is possible for the lines to saturate at $85 \%$ of the continuum even with small covering factors (e.g., $24 \%$ and $6 \%$ for $\mathrm{C}_{2} \mathrm{H}_{2}$ ) since the line widths are narrower than our spectral resolution. Presumably the same statement applies to the other observed molecules. 


\section{3. $\mathrm{NH}_{3}$}

From our infrared observations, we find that $\mathrm{NH}_{3}$ has two components: one at $-57.3 \mathrm{~km} \mathrm{~s}^{-1}$ and the other at $-60.1 \mathrm{~km}$ $\mathrm{s}^{-1}$. Most of the $\mathrm{NH}_{3}$ is found in the first component, for which the temperature is $\sim 278 \mathrm{~K}$ and the column density is $5.2 \times 10^{16} \mathrm{~cm}^{-2}$. The second component has a lower temperature $(\sim 248 \mathrm{~K})$ and the column density is $2.8 \times 10^{16} \mathrm{~cm}^{-2}$ (see Table 3). $\mathrm{NH}_{3}$ absorption toward IRS 1 has also been studied in the radio by Wilson et al. (1983) and Henkel et al. (1984). They found $N_{\mathrm{NH}_{3}}=2 \times 10^{18} \mathrm{~cm}^{-2}$ in gas with $T=170$ $220 \mathrm{~K}$ and $V_{\mathrm{LSR}}=-60 \mathrm{~km} \mathrm{~s}^{-1}$. Although their temperature and Doppler shift is in reasonable agreement with our bluer absorption component, their column density is nearly 20 times ours. Without constraining the ${ }^{15} \mathrm{~N} /{ }^{14} \mathrm{~N}$ isotopic ratio, our data allow an $\mathrm{NH}_{3}$ column as large as that from radio observations, but with this $\mathrm{NH}_{3}$ column ${ }^{15} \mathrm{NH}_{3}$ lines should have been apparent in our spectra. It is possible that we sample only a fraction of the column observed at $\mathrm{cm}$ wavelengths as a result of larger dust opacity at mid-infrared wavelengths preventing us from observing lines of sight through the densest regions of a knotty gas distribution. It may be notable that Wilson et al. found, as did we, that although relative depths of lines (in their case hyperfine components) require large optical depths, line shapes do not appear flat-bottomed as would be expected if they are optically thick. They suggest that the absorbing gas may be in small spectrally and spatially unresolved knots, each of which is optically thick, and which combine to make a line that does not have the shape of a thick line. Our use of two components in our fit may be a way of approximating this situation.

\section{4. $\mathrm{HNCO}$}

The velocities for the two components are -57.2 and $-60.2 \mathrm{~km} \mathrm{~s}^{-1}$, with corresponding temperatures of 319 and $171 \mathrm{~K}$ and column densities of $4 \times 10^{15}$ and $1 \times 10^{15} \mathrm{~cm}^{-2}$. Since the HNCO temperatures and velocities derived from our observations agree with the values for the other molecules, we can assume that HNCO is in the same gas. It appears the region probed by $\mathrm{HNCO}$ is very small in angular size. Zinchenko et al. (2000) detect HNCO emission in the radio and derive a beam-averaged column density of $1.1 \times 10^{14} \mathrm{~cm}^{-2}$. This value is an order of magnitude lower than our observed column density. However, beam dilution in the radio observations can explain the difference in the column densities.

\section{5. $\mathrm{CH}_{3}$}

$\mathrm{CH}_{3}$ is detected for the first time toward warm, dense gas. The parameters derived for this molecule do not agree as well with the other molecules, although the weakness of the observed lines makes the derived parameters rather uncertain. The separation between the centroid velocities for the two components, $V_{\mathrm{LSR}}=-54.2$ and -62.8 , is nearly twice the separation found for the other molecules. Also, the covering factor for both of the components is consistent with $100 \%$, whereas as it is closer to $10 \%$ for the other molecules. In addition, the temperature of the second component is much hotter (greater than $900 \mathrm{~K}$ ) than the temperatures derived for the other molecules. We note though that the fact that the observed lines are of high excitation makes them particularly sensitive to a hot gas component, which may not have been noticed for other molecules. And the weakness of the lines makes the derived covering factor (which is inferred from relative line depths indicating saturation) very uncertain.

\section{6. $\mathrm{CH}_{4}$ and $\mathrm{CS}$}

$\mathrm{CH}_{4}$ gas-phase absorption had been observed toward the neighboring protostar IRS 9 (Lacy et al. 1991; Boogert et al. 2004) but had not previously been seen toward IRS 1 . We find centroid velocities of -56.3 and $-60.1 \mathrm{~km} \mathrm{~s}^{-1}$, which are similar to the other molecules. The temperatures are higher than for most molecules ( $\sim 670 \mathrm{~K}$ for both components) but have large uncertainties (see Table 3 ). The best fit seems to indicate that one of the components covers the entire source whereas the other covers about $6 \%$ of the source. Of all of the observed molecules, $\mathrm{CH}_{4}$ has the highest column density. The implications of the high $\mathrm{CH}_{4}$ column density the chemistry are discussed in Section 5.1.

We detect only six lines of CS (see Table 2-full table available online only). The lines are rather weak and parameters are poorly constrained. Despite uncertainties, the parameters agree with those for other molecules. The centroid velocities are at -55.2 and $-59.4 \mathrm{~km} \mathrm{~s}^{-1}$. The temperatures, $T_{1}=224 \mathrm{~K}$ and $T_{2}=249 \mathrm{~K}$, are similar to those found other molecules (except for $\mathrm{CH}_{4}$ and $\mathrm{CH}_{3}$ ). From radio studies of the envelope around IRS 1, a CS abundance of $\sim 10^{-10}$ is found (Plume et al. 1997; Mueller et al. 2002; Shirley et al. 2003). However, also using radio observations, van der Tak et al. (2000) derive a CS abundance of $\sim 10^{-8}$. Our abundance is about 2 orders of magnitude larger than the largest estimate of the abundance in the envelope indicating that our observations are probing material closer to the protostar.

\section{MODELS}

\subsection{Chemical Models}

We now consider the implications of the observations for the chemistry of the observed material. Hot core models can be used to represent two of the scenarios discussed in Section 1: (1) material in a circumstellar disk and (2) photoevaporating knots of neutral molecular material. Hot cores are small $(r<0.1 \mathrm{pc})$, dense $\left(n_{\mathrm{H}_{2}}>10^{7} \mathrm{~cm}^{-3}\right)$ and hot $(T>100 \mathrm{~K})$ regions associated with high-mass young stellar objects (Kurtz et al. 2000). Chemically, hot cores are identified by an enhanced abundance of fully hydrogenated molecules such as $\mathrm{H}_{2} \mathrm{O}$ and $\mathrm{NH}_{3}$, which are usually observed through rotational transitions at submillimeter and millimeter wavelengths. These abundances are enhanced with respect to the cold molecular envelope. A hot core is thought to form as a result of the protostar heating nearby material, which triggers ice sublimation from the grain mantles (Charnley et al. 1992). HNCO is another hot core molecule which has been found in the Orion hot core with an abundance $\sim 10^{-8}$ relative to $\mathrm{H}_{2}$ (Zinchenko et al. 2000).

Gas-phase HNCO is possibly coming from evaporation of grain mantles. The $4.62 \mu \mathrm{m}$ feature observed toward some protostars has been attributed to $\mathrm{OCN}^{-}$frozen in grain mantles (e.g., Pendleton et al. 1999; van Broekhuizen et al. 2004). Recent $I S O$ results of solid state features toward protostars show an $\mathrm{OCN}^{-}$upper limit of $10^{16} \mathrm{~cm}^{-2}$ toward IRS 1 (Gibb et al. 2004). The total HNCO column derived in this work is $5.4 \times 10^{15}$ $\mathrm{cm}^{-2}$, a factor of 2 lower than the solid $\mathrm{OCN}^{-}$limit. In general, IRS 1 does not show many ice features compared to the colder nearby source IRS 9 (see Gibb et al. 2004), which has a column density $N\left(\mathrm{OCN}^{-}\right)=1.2 \times 10^{17} \mathrm{~cm}^{-2}$. If solid $\mathrm{OCN}^{-}$sublimates and captures a proton to form $\mathrm{HNCO}$, the expected column density for $\mathrm{HNCO}$ would be comparable to the observed $\mathrm{OCN}^{-}$ ice toward cold lines of sight (like IRS 9). However, the gasphase column observed is at least an order of magnitude smaller 
Table 5

$N(\mathrm{X}) / N(\mathrm{CO})$ from Chemical Models

\begin{tabular}{lccc}
\hline \hline Molecule & $\begin{array}{c}\text { Observed } \\
\left(10^{-3}\right)\end{array}$ & $\begin{array}{c}\text { Hot Core Model } \\
\left(10^{-3}\right)\end{array}$ & $\begin{array}{c}\text { Disk Models }^{\mathrm{b}} \\
\left(10^{-3}\right)\end{array}$ \\
\hline $\mathrm{C}_{2} \mathrm{H}_{2}$ & 5.7 & 0.88 & $0.06-0.1$ \\
$\mathrm{HCN}$ & 6.9 & 0.02 & $16-24$ \\
$\mathrm{CH}_{3}$ & 2.3 & 0.016 & $0.02-1.6$ \\
$\mathrm{NH}_{3}$ & 8.1 & 0.76 & $1-6$ \\
$\mathrm{CH}_{4}$ & 36 & 1.6 & $7-35$ \\
$\mathrm{CS}^{*}$ & 7.0 & 0.0031 & $0.03-0.07$
\end{tabular}

Notes. The model values were taken at $10^{4}$ years.

${ }^{a}$ Nomura \& Millar (2004) and H. Nomura (2008, private communication).

${ }^{b}$ Nguyen et al. (2002).

than that of the solid state ion toward sources like IRS 9. This indicates that when $\mathrm{OCN}^{-}$sublimates, a fraction of the ices goes into forming $\mathrm{HNCO}$, while most of $\mathrm{HNCO}$ is destroyed in the warm gas-phase chemistry on short time scales. Further studies of how $\mathrm{OCN}^{-}$reacts in the gas-phase are needed in order to understand the difference between ice and gas phase column densities.

Similarly, $\mathrm{CH}_{4}$ is probably also evaporating from the grain mantles, and thus the gas-phase abundance is highly enhanced compared with the cold molecular envelope. Studies of the solid $\mathrm{CH}_{4}$ content toward IRS 1 show that the column density is 14 times less than our observed gas-phase column (Gibb et al. 2004), suggesting that the solid $\mathrm{CH}_{4}$ on grains has sublimated. In comparison, IRS 9 has a higher content of solid $\mathrm{CH}_{4}$ than gas-phase $\mathrm{CH}_{4}$ (Boogert et al. 2004; Gibb et al. 2004). If we consider that IRS 1 is likely a more evolved object than IRS 9 (Elmegreen \& Lada 1977), the higher gas-phase $\mathrm{CH}_{4}$ toward IRS 1 also points to grain mantle evaporation. The high abundance of $\mathrm{CH}_{4}$ means that daughter products such as $\mathrm{CH}_{3}$ and $\mathrm{CH}_{2}$ should be abundant. While the photodissociation of $\mathrm{CH}_{4}$ preferentially forms $\mathrm{CH}_{2}$ rather than $\mathrm{CH}_{3}$, our observations of $\mathrm{CH}_{3}$ agree with this branching ratio. We predict that $\mathrm{CH}_{2}$ is also present toward IRS 1 with a higher abundance than $\mathrm{CH}_{3}$. It is uncertain where $\mathrm{C}_{2} \mathrm{H}_{2}$ is formed in the gas-phase chemistry or is evaporating from grain mantles. However, most models do not predict the observed column densities from formation in gas-phase chemistry only.

From our observations we see a high abundance of the parent molecules $\mathrm{NH}_{3}$ and $\mathrm{CH}_{4}$. High abundances of the other molecules are also observed. Nomura \& Millar (2004) present a time dependent chemical evolution of molecules in a hot core. The models begin at the time when the protostar turns on $(t=0$ years). Table 5 shows the abundances with respect to $\mathrm{CO}$ at an age of $10^{4}$ years. The hot core model seems to underpredict abundances with respect to $\mathrm{CO}$ of all our observed molecules. However, as mentioned in Section 4.2, the CO column density found by Mitchell et al. (1990) may not be the appropriate number with which to compare our observations. If the CO column along the lines of sight containing our molecules is larger than the column measured by Mitchell et al., then perhaps the abundances from the model will more closely resemble the observations. We can also compare the relative abundances between our observed molecules, avoiding the uncertainties in whether the $\mathrm{CO}$ and dust absorption trace the lines of sight that our molecules are found in. The observed and predicted values for $N\left(\mathrm{CH}_{3}\right) / N\left(\mathrm{CH}_{4}\right)$ are similar, $\sim 0.01$. For the other molecules the ratios from the models at an age of $10^{4}$ years are very different from the observed values. The values for the models assume that we can see all the material

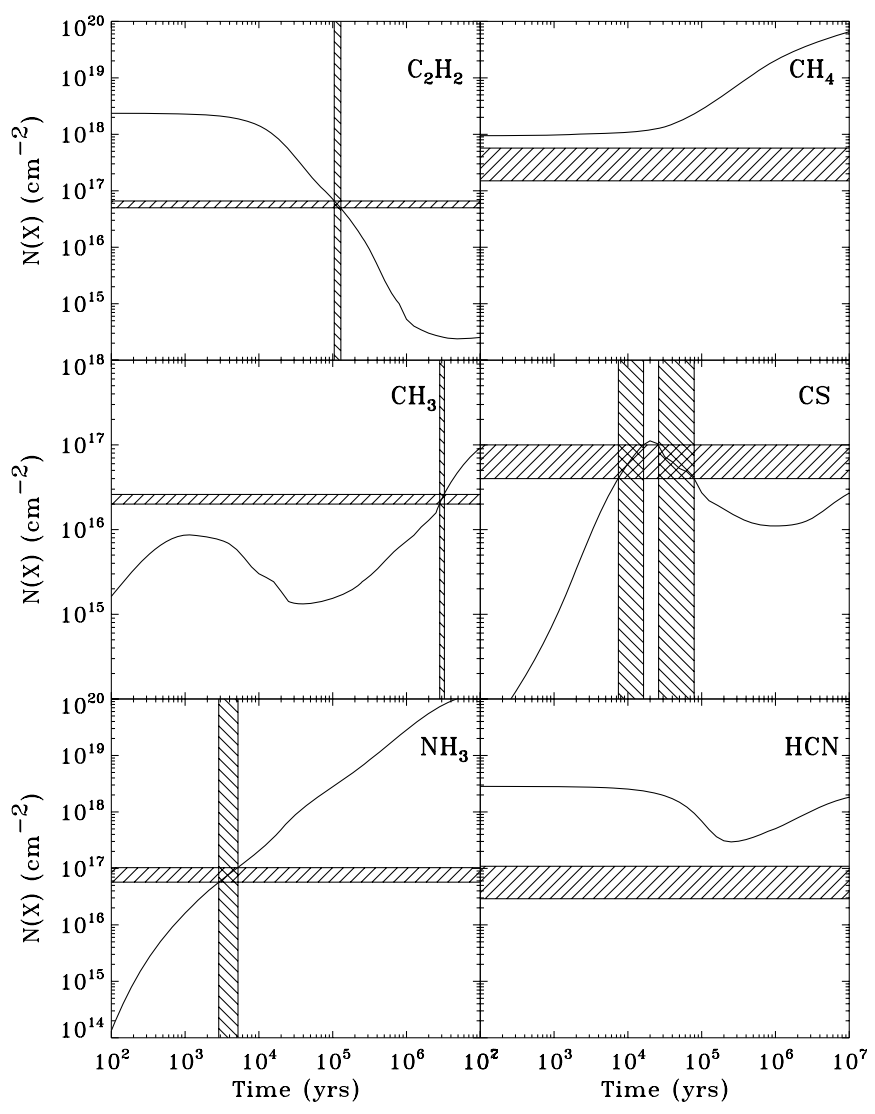

Figure 7. These plots illustrate the determination of ages based on the column densities of the various molecules compared with time dependent chemical model. The model data are from Nomura \& Millar (2004). The horizontal lines indicate the range in column densities derived in this paper. The vertical lines indicate the age range corresponding to those column densities. Note that for $\mathrm{CH}_{4}$ and $\mathrm{HCN}$ the model overpredicts the column density at all ages.

and comparing the model results directly with the observations without accounting for line-of-sight does not make for a good comparison. Figure 7 shows the column density evolution for six of the detected molecules as determined by Nomura \& Millar (2004) corrected for the observed beam (H. Nomura 2008 , private communication). For each molecule, the observed range of column densities is shown in the horizontal shaded area. This hot core model can predict the observed column densities for four of the six molecules plotted. However, the ages indicated by each molecule do not give a consistent overall age. For $\mathrm{CH}_{4}$ and $\mathrm{HCN}$ the model overpredicts the observed column densities. The other molecules suggest ages between $2 \times 10^{3}$ and $2 \times 10^{6}$ years. While ages vary by three orders of magnitude, it should be noted that the model has not been optimized to be an accurate representation of this source. With chemical models that more closely represent the source, it may be possible to determine a more consistent age.

If we consider the disk scenario, we can also compare our observations to a chemical model of a photoevaporating disk. Nguyen et al. (2002) present a chemical model for a disk around a $10 M_{\odot}$ star. The model consists of a flared disk with a photoevaporating layer on the surface. Table 5 compares our derived abundances with respect to $\mathrm{CO}$ for the various molecules to the predicted values from disk models. The summarized results of models taking the midplane temperature for the temperature of the layers below the photodissociation region (PDR) are discussed here. The difference among the three models is the variation in the X-ray ionization rate, $\zeta$ 


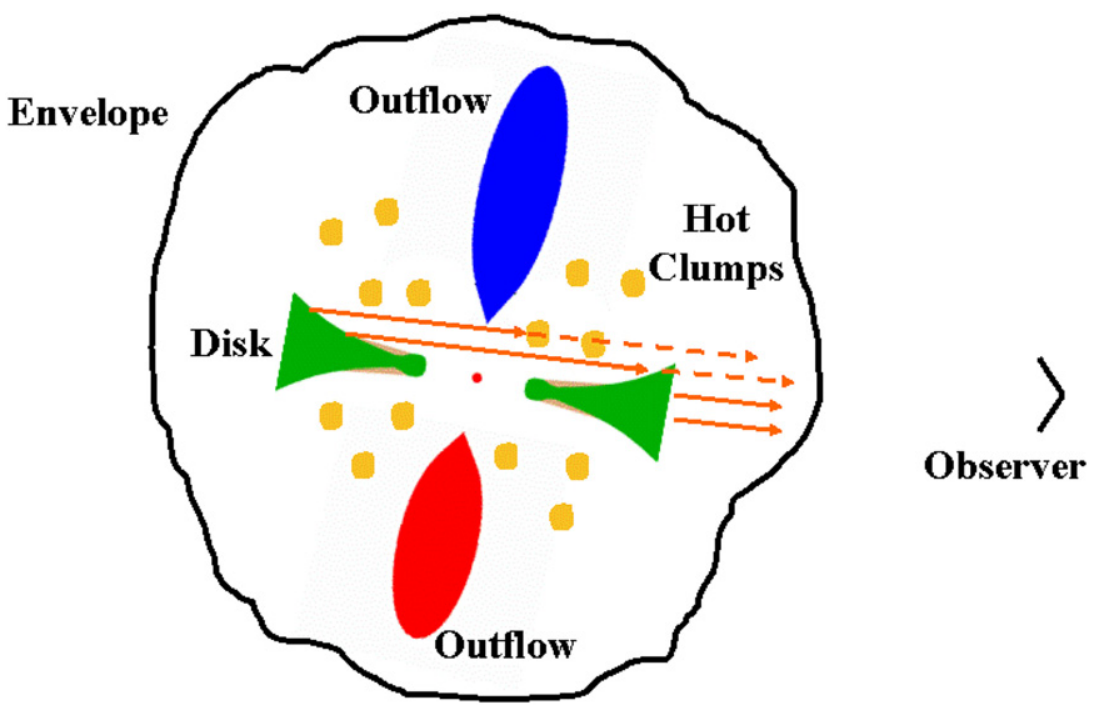

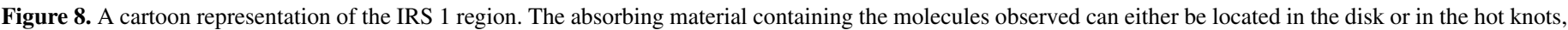

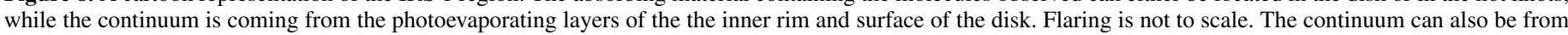

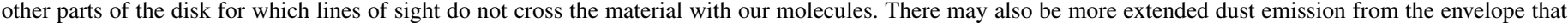
contributes to the continuum but does not contain our molecules in high abundances. In this figure, North roughly points toward the top of the page.

(A color version of this figure is available in the online journal.)

$\left(1 \zeta=1.3 \times 10^{-17} \mathrm{~s}^{-1}\right)$, from 1 to $10^{5} \zeta$. Table 5 shows that the abundance for $\mathrm{C}_{2} \mathrm{H}_{2}$ is underpredicted. In the chemical model, $\mathrm{C}_{2} \mathrm{H}_{2}$ is produced only by gas phase reactions. One possible explanation is that $\mathrm{C}_{2} \mathrm{H}_{2}$ is formed as ice on grain mantles. The abundance of $\mathrm{NH}_{3}$ is also underpredicted. The model with the highest X-ray ionization rate produces $\mathrm{CH}_{3}$ abundances close to the observed value. For that model, $N\left(\mathrm{CH}_{3}\right) / N\left(\mathrm{CH}_{4}\right)$ is 4 times larger than the observed ratio. These models do not fit our observations very well but can serve as a guide to the expected abundances in such a scenario. The model by Nguyen et al. has not been modified to try to fit data. So, adapting the model for this object may give predictions closer to the observations. The star in NGC 7538 IRS 1 is believed to be a $30 M_{\odot}$ star. The different radiation field may affect the resulting chemistry.

Doty et al. (2002) present a model of chemical evolution of the envelopes of massive protostars using AFGL 2591 as an example. From that model, they conclude that enhancements of $\mathrm{C}_{2} \mathrm{H}_{2}$, $\mathrm{HCN}$, and $\mathrm{CH}_{4}$ are possible at late times in the evolution (greater than $10^{5}$ years) and at high temperature $(T \sim 800 \mathrm{~K})$. Our derived temperatures indicate cooler material, yet the molecules seem to be enhanced in the line of sight. Alternatively, these species could be produced at high temperature in the inner disk, and subsequently brought outward by the disk wind. Including UV and X-ray radiation in the model affects the chemistry especially relating to $\mathrm{HCN}$. Stäuber et al. $(2004,2005)$ find that increasing the UV and X-ray flux can lead to enhanced HCN starting by the ionization of $\mathrm{N}_{2}$. The column densities for $\mathrm{HCN}$ and CS from the Stäuber et al. model of protostar AFGL 2591 agree with our observed abundances while their column densities for $\mathrm{C}_{2} \mathrm{H}_{2}, \mathrm{CH}_{4}$, and $\mathrm{NH}_{3}$ are lower than our observed values.

Hot core and disk chemistry models predict the enhancement of molecules such as $\mathrm{C}_{2} \mathrm{H}_{2}, \mathrm{CH}_{4}$, and $\mathrm{NH}_{3}$. However, the observed abundance of $\mathrm{C}_{2} \mathrm{H}_{2}$ is higher than what models predict from warm gas-phase chemistry (see Table 5). This indicates that $\mathrm{C}_{2} \mathrm{H}_{2}$ is probably frozen on dust grains and sublimates along with molecules like $\mathrm{CH}_{4}$. Boudin et al. (1998) studied the solid features of $\mathrm{C}_{2} \mathrm{H}_{2}$, especially when mixed with $\mathrm{H}_{2} \mathrm{O}$ or $\mathrm{CO}$. They found that the $\mathrm{C}_{2} \mathrm{H}_{2}$ features broaden substantially when mixed with $\mathrm{H}_{2} \mathrm{O}$ and to a lesser extent when mixed with $\mathrm{CO}$. They compare the laboratory data to ISO data for the colder neighbor, IRS 9, which resulted in an upper limit of $8 \times 10^{17} \mathrm{~cm}^{-2}$ for the column density of solid $\mathrm{C}_{2} \mathrm{H}_{2}$. This upper limit is consistent with the observed gas-phase column density seen toward IRS 1. So, it is possible that solid $\mathrm{C}_{2} \mathrm{H}_{2}$ is sublimating from grain mantles as protostars heat the environment. The observed $\mathrm{CH}_{3} / \mathrm{CH}_{4}$ ratio agrees with the branching ratio for the destruction of $\mathrm{CH}_{4}$. Based on these results $\mathrm{CH}_{2}$ should also be very abundant.

\subsection{Physical Models}

We now attempt to construct a physical and geometrical model of NGC 7538 IRS 1. Within the possible scenarios presented from various radio and infrared observations (e.g., Minier et al. 2001; Lugo et al. 2004; De Buizer \& Minier 2005; Kraus et al. 2006), we propose a scenario in which the molecular absorption presented here comes from a circumstellar disk. We will examine other possibilities first. Figure 8 depicts the possible scenarios allowed by the available observations.

We can quickly rule out a simple model in which the absorbing molecular gas is in the foreground molecular cloud and not closely associated with IRS 1 . Our observations require the gas to be much hotter $(T \sim 300 \mathrm{~K})$ and denser $\left(n_{\mathrm{H}_{2}} \sim 10^{7} \mathrm{~cm}^{-3}\right.$ to maintain rotational LTE of HCN out to $J=21$ ) than is found away from luminous sources in molecular clouds. According to van der Tak et al. (2000), temperatures do not reach the observed values until you get to within $400 \mathrm{AU}$ of the central star. In addition, the infrared radiation field must have a brightness temperature $\sim 300 \mathrm{~K}$ to populate the $\mathrm{C}_{2} \mathrm{H}_{2} \nu_{5}$ level sufficiently to account for the observed $2 v_{5}-v_{5}$ absorption. Undoubtedly, the absorbing molecular gas is in close proximity to the infrared continuum source IRS 1 .

It is not quite so easy to rule out a model in which the absorbing molecules are in boundary region between the envelope around the IRS 1 hypercompact $\mathrm{H}$ II region and the outflow. This gas could be compressed by the ionized wind, and if it is as close as $0^{\prime \prime} 1$, or $280 \mathrm{AU}$, from a $10^{5} L_{\odot}$ source it would have a temperature near $300 \mathrm{~K}$. The temperature structure determined by van der Tak et al. (2000) indicates that indeed temperatures 
range from 200 to $400 \mathrm{~K}$ at radii of 220-380 AU. However, interaction with the ionized wind, which has a velocity $\sim 100 \mathrm{~km} \mathrm{~s}^{-1}$, would be expected to accelerate the gas, causing broad, blue-shifted absorption (see van der Tak et al. 2000). In contrast, the observed lines have widths of $<8 \mathrm{~km} \mathrm{~s}^{-1}$ and have centroids within a few $\mathrm{km} \mathrm{s}^{-1}$ of those seen in surrounding molecular gas. Another argument against the presence of the observed gas in an envelope around IRS 1 is the fact that net absorption is seen. Since the vibrational temperature is comparable to the brightness temperature of the continuum radiation, emission lines would be seen if the molecular gas had a larger extent than the continuum source. If the lines arose in molecular shell surrounding the hypercompact $\mathrm{H}$ II region, this probably would be the case.

On the other hand, Campbell (1984) proposes that the centimeter continuum from IRS 1 results from partially ionized material in an outflow. The centimeter continuum emission has been spatially resolved into knots by Gaume et al. (1995), who suggest that the emission comes from photoevaporation of knots of neutral molecular material (see Figure 8). The $\mathrm{CH}_{3} \mathrm{OH}$ masers seen by Minier et al. (2000) also seem to trace the knotty structure (in addition to the disk described in Section 1) probed by the $\mathrm{cm}$ continuum emission. The knots may be material stripped from the disk. The stellar wind would then blow on these knots. However, if our molecules are in these knots we would expect large velocity difference between the knots resulting in broad lines, which are not observed. If we compare the velocities we derive for the two components of our molecules to those observed for the $\mathrm{CH}_{3} \mathrm{OH}$ masers, we find that they match the two velocities found for the A cluster of masers. This indicates that the infrared observations may be probing the same gas as the $\mathrm{CH}_{3} \mathrm{OH}$ masers. This scenario is further supported by Kraus et al. (2006). However, Kraus et al. also find that the interpretation of the masers tracing a disk also agrees with their observations.

We propose that the absorbing material is in a circumstellar disk. There is evidence for a disk close to edge-on from other observations and models (e.g., Pestalozzi et al. 2004; Lugo et al. 2004; De Buizer \& Minier 2005). In this situation, hot dust in the surface layers or the inner rim of a flared disk could provide the continuum source. With a nearly edge-on orientation, radiation from the inner region could pass through the outer disk atmosphere, where absorption lines could be formed. Emission from some of the dust on the surface of a slightly inclined disk or in an outflow could be observed directly, accounting for the dilution of the spectrum that we modeled with a small covering factor. This scenario is similar to the one described by Lahuis et al. (2006) to describe $\mathrm{C}_{2} \mathrm{H}_{2}$ and $\mathrm{HCN}$ absorption toward the low-mass protostar IRS 46 in Ophiuchus (YLW 16B). Detailed modeling of the disk structure of IRS 46 showed that it was possible to have the absorbing material in the disk illuminated by the continuum from the surface. Figure 8 is a cartoon of our proposed picture, where the orange rays represent the continuum. Some of the continuum radiation passes through the absorbing outer disk. In this scenario, dust settling is likely to occur, allowing for observations of large columns of gas without too much dust.

\section{CONCLUSIONS}

Here we present a summary of our conclusions.

1. NGC 7538IRS 1 has a rich spectrum in the mid-infrared. At high spectral resolution, lines of previously observed molecules such as $\mathrm{C}_{2} \mathrm{H}_{2}$ and $\mathrm{HCN}$ are seen as well as weak lines of molecules such as $\mathrm{HNCO}, \mathrm{CH}_{3}, \mathrm{NH}_{3}, \mathrm{CH}_{4}$, and $\mathrm{CS}$. We have presented the first infrared detection of interstellar $\mathrm{HNCO}$. This is also the first detection of $\mathrm{CH}_{3}$ toward dense gas.

2. The data show shallow yet saturated lines indicating that either the absorbing gas does not fully cover the continuum source or there is emission filling in the absorption. The observed ortho:para equivalent width ratio for $\mathrm{C}_{2} \mathrm{H}_{2}$ is $\sim 1.5$ instead of the expected value of 3 .

3. The rotational temperatures for the various molecules range between 200 and $400 \mathrm{~K}$ with the exception of $\mathrm{CH}_{3}$, which has one hot component $(T \sim 900 \mathrm{~K})$. From the derived temperatures, we find that the gas is within $\sim 400$ AU of the central star (van der Tak et al. 2000).

4. We suggest that the material traces a close to edgeon circumstellar disk as determined from other observations (e.g., Minier et al. 1998). However, it can also be tracing hot, knotty material close in to the star (see Figure 8).

5. Because it is possible to use hot core chemistry to describe chemistry in a disk, the different chemical models do not help identify the physical location of the absorbing gas. However, the models do seem to indicate that NGC 7538 IRS 1 is an evolved protostar with an age $\sim 10^{5}$ years.

6. Our observations help constrain the chemistry in massive protostars. The abundances for all the molecules are enhanced, except for possibly CS (see discussion in Section 4.6). Species known to be present in icy mantles such as $\mathrm{CH}_{4}$ and $\mathrm{NH}_{3}$ have high column densities suggesting that they have recently sublimated from grain mantles. The high abundance of the daughter molecules such $\mathrm{CH}_{3}$ gives constraints on the gas-phase chemistry happening after sublimation of ices. HNCO also provides some constraints about the presence of $\mathrm{OCN}^{-}$on dust grains. It is unclear whether $\mathrm{C}_{2} \mathrm{H}_{2}$ is a parent molecule or a product of warm gas-phase chemistry. For molecules like HCN, UV/X-ray radiation is important in producing the observed abundances.

C.K. thanks A.C.A. Boogert, A.M.S. Boonman-Gloudemans, S. Doty, and F. Lahuis for useful discussions. The authors thank T.K. Greathouse for assisting with the observations. C.K. acknowledges support from the NASA Astrobiology Program under RTOP-344-53-51. This work was partly supported by NSF grant AST-0607312.

\section{APPENDIX \\ SPECTROSCOPY OF $\mathrm{CH}_{3}$ AND HNCO}

$\mathrm{CH}_{3}$ is a nearly planar molecule and thus we do not expect to see inversion transitions as observed in $\mathrm{NH}_{3}$. We observe the out-of-plane vibrational mode (similar to the umbrella mode in $\mathrm{NH}_{3}$ ). We observe splitting due to spin-rotation interactions. For symmetric-top molecules, the splitting of a rotational level is given by

$$
\Delta \nu=(N+1 / 2)\left\{\epsilon_{b b}^{(v)}-\left(\epsilon_{b b}^{(v)}-\epsilon_{c c}^{(v)}\right) K^{2} /[N(N+1)]\right\},
$$

where $\epsilon_{b b}^{(\nu)}$ and $\epsilon_{c c}^{(v)}$ are the spin-rotation coupling constants for the $\mathrm{B}$ and $\mathrm{C}$ axes, respectively, at a given vibrational state, $v$. We use Equation (A1) in deriving the splitting for the lines we observed. The values for $\epsilon_{b b}^{(v)}$ and $\epsilon_{c c}^{(v)}$ were taken from Yamada et al. (1981). 
Table 6

HNCO Selection Rules

\begin{tabular}{lclc}
\hline \hline \multicolumn{2}{c}{$\Delta K=0$} & \multicolumn{2}{c}{$\Delta K= \pm 1$} \\
\hline$K \neq 0$ & & & \\
$\Delta J=0$ & $\Delta J= \pm 1$ & $\Delta J=0$ & $\Delta J= \pm 1$ \\
$U \leftrightarrow L$ & $U \leftrightarrow U$ & $U \leftrightarrow U$ & $U \leftrightarrow L$ \\
& $L \leftrightarrow L$ & $L \leftrightarrow L$ & \\
\hline
\end{tabular}

HNCO is a quasi-linear, nearly symmetric-top molecule. The moment of inertia about the figure axis is small and the moments of inertia about the other two axes are much larger $(\sim 100$ times larger) and about equal to each other. Because it is a slightly asymmetric molecule, the levels with $K \neq 0$ are split into two components. Steiner et al. (1979) refer to these levels as upper $(\mathrm{U})$ and lower $(\mathrm{L})$. Table 6 shows the selection rules for HNCO.

In Figure 4, the HNCO lines labeled $\mathrm{R}(\mathrm{J}) 1 \mathrm{U}$ correspond to lines where $\Delta K=0$ and $\Delta J=1$ and $K=1$ upper. Likewise, the lines labeled $\mathrm{R}(\mathrm{J}) 1 \mathrm{~L}$ are lines where $\Delta K=0$ and $\Delta J=1$ and $K=1$ lower. Because the splitting between the two levels is small the lines overlap in the spectral region shown in Figures 3 and 4. Some lines from the upper and lower K states are blended together and give the appearance of double peaked lines.

\section{REFERENCES}

Bevington, P. R., \& Robinson, D. K. 2003, Data Reduction and Error Analysis for the Physical Sciences (3rd ed.; Boston, MA: McGraw-Hill)

Boogert, A. C. A., Blake, G. A., \& Öberg, K. 2004, ApJ, 615, 344

Boonman, A. M. S., \& van Dishoeck, E. F. 2003, A\&A, 403, 1003

Boonman, A. M. S., van Dishoeck, E. F., Lahuis, F., \& Doty, S. D. 2003, A\&A, 399, 1063

Botschwina, P., \& Sebald, P. 1985, J. Mol. Spectrosc., 110, 1

Boudin, N., Schutte, W. A., \& Greenberg, J. M. 1998, A\&A, 331, 749

Campbell, B. 1984, ApJ, 282, L27

Campbell, B., \& Thompson, R. I. 1984, ApJ, 279, 650

Charnley, S. B., Tielens, A. G. G. M., \& Millar, T. J. 1992, ApJ, 399, L71

De Buizer, J. M., \& Minier, V. 2005, ApJ, 628, L151

Dickel, H. R., Rots, A. H., Goss, W. M., \& Forster, J. R. 1982, MNRAS, 198, 265

Doty, S. D., van Dishoeck, E. F., van der Tak, F. F. S., \& Boonman, A. M. S. 2002, A\&A, 389, 446

Elmegreen, B. G., \& Lada, C. J. 1977, ApJ, 214, 725

Feuchtgruber, H., Helmich, F. P., van Dishoeck, E. F., \& Wright, C. M. 2000, ApJ, 535, L111

Gaume, R. A., Goss, W. M., Dickel, H. R., Wilson, T. L., \& Johnston, K. J. 1995, ApJ, 438, 776

Gibb, E. L., Whittet, D. C. B., Boogert, A. C. A., \& Tielens, A. G. G. M. 2004, ApJS, 151, 35

Henkel, C., Wilson, T. L., \& Johnston, K. J. 1984, ApJ, 282, L93

Hoffman, I. M., Goss, W. M., Palmer, P., \& Richards, A. M. S. 2003, ApJ, 598, 1061

Kameya, O., Morita, K. I., Kawabe, R., \& Ishiguro, M. 1990, ApJ, 355, 562

Kraus, S., et al. 2006, A\&A, 1000, 446
Kurtz, S., Cesaroni, R., Churchwell, E., Hofner, P., \& Walmsley, C. M. 2000, in Protostars and Planets IV, ed. V. Mannings, A. P. Boss, \& S. S. Russell (Tucson, AZ: Univ. Arizona Press), 299

Lacy, J. H., Achtermann, J. M., Bruce, D. E., Lester, D. F., Arens, J. F., Peck, M. C., \& Gaalema, S. D. 1989, PASP, 101, 1166

Lacy, J. H., Carr, J. S., Evans, N. J., Baas, F., Achtermann, J. M., \& Arens, J. F. 1991, ApJ, 376, 556

Lacy, J. H., Richter, M. J., Greathouse, T. K., Jaffe, D. T., \& Zhu, Q. 2002, PASP 114,153

Lahuis, F., \& van Dishoeck, E. F. 2000, A\&A, 355, 699

Lahuis, F., et al. 2006, ApJ, 636, L145

Lowenthal, M. S., Khanna, R. K., \& Moore, M. H. 2002, Spectrochim. Acta A, 58,73

Lugo, J., Lizano, S., \& Garay, G. 2004, ApJ, 614, 807

Madden, S. C., Irvine, W. M., Matthews, H. E., Brown, R. D., \& Godfrey, P. D. 1986, ApJ, 300, L79

Martin, A. H. M. 1973, MNRAS, 163, 141

Menten, K. M., Walmsley, C. M., Henkel, C., Wilson, T. L., Snyder, L. E., Hollis, J. M., \& Lovas, F. J. 1986, A\&A, 169, 271

Minier, V., Booth, R. S., \& Conway, J. E. 1998, A\&A, 336, L5

Minier, V., Booth, R. S., \& Conway, J. E. 2000, A\&A, 362, 1093

Minier, V., Conway, J. E., \& Booth, R. S. 2001, A\&A, 369, 278

Mitchell, G. F., Maillard, J.-P., Allen, M., Beer, R., \& Belcourt, K. 1990, ApJ, 363,554

Moscadelli, L., Reid, M. J., Menten, K. M., Brunthaler, A., Zheng, X. W., \& Xu, Y. 2009, ApJ, 693, 406

Mueller, K. E., Shirley, Y. L., Evans, N. J., \& Jacobson, H. R. 2002, ApJS, 143, 469

Nguyen, T. K., Viti, S., \& Williams, D. A. 2002, A\&A, 387, 1083

Nomura, H., \& Millar, T. J. 2004, A\&A, 414, 409

Pendleton, Y. J., Tielens, A. G. G. M., Tokunaga, A. T., \& Bernstein, M. P. 1999, ApJ, 513, 294

Pestalozzi, M. R., Elitzur, M., Conway, J. E., \& Booth, R. S. 2004, ApJ, 603, L113

Plume, R., Jaffe, D. T., Evans, N. J., Martin-Pintado, J., \& Gomez-Gonzalez, J. 1997, ApJ, 476, 730

Pratap, P., Batrla, W., \& Snyder, L. E. 1989, ApJ, 341, 832

Scoville, N. Z., Sargent, A. I., Sanders, D. B., Claussen, M. J., Masson, C. R., Lo, K. Y., \& Phillips, T. G. 1986, ApJ, 303, 416

Shirley, Y. L., Evans, N. J., Young, K. E., Knez, C., \& Jaffe, D. T. 2003, ApJS, 149,375

Stäuber, P., Doty, S. D., van Dishoeck, E. F., \& Benz, A. O. 2005, A\&A, 440, 949

Stäuber, P., Doty, S. D., van Dishoeck, E. F., Jørgensen, J. K., \& Benz, A. O 2004, A\&A, 425, 577

Steiner, D. A., Wishah, K. A., Polo, S. R., \& McCubbin, T. K. 1979, J. Mol Spectrosc., 76, 341

Ungerechts, H., Umbanhowar, P., \& Thaddeus, P. 2000, ApJ, 537, 221

van Broekhuizen, F. A., Keane, J. V., \& Schutte, W. A. 2004, A\&A, 415, 425

van der Tak, F. F. S., van Dishoeck, E. F., Evans, N. J., \& Blake, G. A. 2000, ApJ, 537, 283

Werner, M. W., Becklin, E. E., Gatley, I., Matthews, K., Neugebauer, G., \& Wynn-Williams, C. G. 1979, MNRAS, 188, 463

Willner, S. P. 1976, ApJ, 206, 728

Willner, S. P., et al. 1982, ApJ, 253, 174

Wilson, T. L., Walmsley, C. M., Batrla, W., \& Mauersberger, R. 1983, A\&A, 127, L19

Wormhoudt, J., \& McCurdy, K. E. 1989, Chem. Phys. Lett., 156, 47

Wynn-Williams, C. G., Becklin, E. E., \& Neugebauer, G. 1974, ApJ, 187, 473

Yamada, C., Hirota, E., \& Kawaguchi, K. 1981, J. Chem. Phys., 75, 5256

Zheng, X.-W., Zhang, Q., Ho, P. T. P., \& Pratap, P. 2001, ApJ, 550, 301

Zinchenko, I., Henkel, C., \& Mao, R. Q. 2000, A\&A, 361, 1079 